\title{
The ALMA View of the OMC1 Explosion in Orion
}

\author{
John Bally ${ }^{1}$, Adam Ginsburg ${ }^{2}$, Hector Arce ${ }^{3}$, Josh Eisner ${ }^{4}$, Allison Youngblood ${ }^{1}$, Luis Zapata ${ }^{5}$, and Hans Zinnecker ${ }^{6}$ \\ ${ }^{1}$ Astrophysical and Planetary Sciences Department University of Colorado, UCB 389 Boulder, Colorado 80309, USA; john.bally@ colorado.edu \\ ${ }^{2}$ ESO Headquarters Karl-Schwarzschild-Str. 2 D-85748, Garching bei Munchen, Germany \\ ${ }^{3}$ Department of Astronomy Steinbach Hall, 52 Hillhouse Avenue, Yale University, New Haven, CT 06511, USA \\ ${ }^{4}$ Steward Observatory University of Arizona, 933 North Cherry Avenue, Tucson, AZ 85721, USA \\ ${ }^{5}$ Instituto de Radioastronomía y Astrofísíca, UNAM Apdo. Postal 3-72 (Xangari), 58089 Morelia, Michoacán, México \\ ${ }^{6}$ Deutsches SOFIA Institut (DSI) University of Stuttgart, Pfaffenwaldring 29, D-70569, Germany \\ Received 2016 September 14; revised 2016 December 7; accepted 2016 December 20; published 2017 March 3
}

\begin{abstract}
Most massive stars form in dense clusters where gravitational interactions with other stars may be common. The two nearest forming massive stars, the BN object and Source I, located behind the Orion Nebula, were ejected with velocities of $\sim 29$ and $\sim 13 \mathrm{~km} \mathrm{~s}^{-1}$ about 500 years ago by such interactions. This event generated an explosion in the gas. New ALMA observations show in unprecedented detail, a roughly spherically symmetric distribution of over a hundred ${ }^{12} \mathrm{CO} J=2-1$ streamers with velocities extending from $V_{\mathrm{LSR}}=-150$ to $+145 \mathrm{~km} \mathrm{~s}^{-1}$. The streamer radial velocities increase (or decrease) linearly with projected distance from the explosion center, forming a "Hubble Flow" confined to within 50" of the explosion center. They point toward the high proper-motion, shockexcited $\mathrm{H}_{2}$ and [Fe II] "fingertips" and lower-velocity $\mathrm{CO}$ in the $\mathrm{H}_{2}$ wakes comprising Orion's "fingers." In some directions, the $\mathrm{H}_{2}$ "fingers" extend more than a factor of two farther from the ejection center than the CO streamers. Such deviations from spherical symmetry may be caused by ejecta running into dense gas or the dynamics of the $\mathrm{N}$-body interaction that ejected the stars and produced the explosion. This $\sim 10^{48} \mathrm{erg}$ event may have been powered by the release of gravitational potential energy associated with the formation of a compact binary or a protostellar merger. Orion may be the prototype for a new class of stellar explosiozn responsible for luminous infrared transients in nearby galaxies.
\end{abstract}

Key words: Herbig-Haro objects - ISM: individual objects (Orion OMC1) - ISM: jets and outflows stars: formation - stars: massive

Supporting material: animation, tar.gz file

\section{Introduction}

Quasi-steady accretion from a circumstellar accretion disk onto forming young stellar objects (YSOs) tends to power long-lived, collimated bipolar outflows and jets common in star-forming regions (Bally \& Reipurth 2001; Frank et al. 2014, p. 451; Bally 2016). Short-lived, powerful explosions, accompanied by luminous infrared flares can be powered by gravitational interactions of three or more stars in dense star clusters that can lead to the formation of binaries by capture and stellar mergers (Bally \& Zinnecker 2005; Soker \& Tylenda 2006; Moeckel \& Bally 2007a, 2007b; Portegies Zwart \& van den Heuvel 2016). Such an explosion occurred behind the Orion Nebula 500 years ago (Zapata et al. 2009; Bally et al. 2011, 2015).

\subsection{Ejection of Runaway Stars: BN, Source I, and Source n}

The $\sim 100 M_{\odot}$ Orion Molecular Core 1 (OMC1) located behind the Nebula (Genzel \& Stutzki 1989) at a distance of $\sim 414$ pc (Menten et al. 2007; Goddi et al. 2011a) is the densest and most molecule-rich part of the degree-long $2200 M_{\odot}$ Integral-Shaped Filament (Johnstone \& Bally 1999) in the $50,000 M_{\odot}$ Orion A cloud (Bally et al. 1987). OMC1 contains the $\sim 1-2 \times 10^{4} L_{\odot}$ but visually obscured Becklin-Neugebauer (BN) object at the north-end of the Kleinmann-Low (KL) infrared nebula $\sim 1^{\prime}$ northwest of the Trapezium stars that ionize the nebula. $\mathrm{BN}$, thought to be an 8 to $15 M_{\odot}$ star (Scoville et al. 1983; Tan 2004; Goddi et al. 2011b; Chatterjee \& Tan 2012), is moving toward the northwest with respect to other stars in the Nebula. The proper motion has been measured to be $21.3 \mathrm{~km} \mathrm{~s}^{-1}$ toward PA $=331^{\circ}$ (Gómez et al. 2008), $26.4 \mathrm{~km} \mathrm{~s}^{-1}$ toward $\mathrm{PA}=341^{\circ}$ (Goddi et al. 2011b), and $26.6 \mathrm{~km} \mathrm{~s}^{-1}$ toward PA $=323^{\circ}$ (Dzib et al. 2016). BN has a redshifted radial velocity of $\sim+11 \mathrm{~km} \mathrm{~s}^{-1}$ with respect to the $V_{\mathrm{LSR}}=+9 \mathrm{~km} \mathrm{~s}^{-1}$ radial velocity of OMC1 (Scoville et al. 1983; Gómez et al. 2008; Goddi et al. 2011b).

It has been proposed that the BN object was ejected by $\theta^{1}$ Ori $\mathrm{C}$, the most massive $\left(\sim 40 M_{\odot}\right)$ member of the Trapezium about 4000 years ago (Tan 2004; Chatterjee \& Tan 2012). However, another massive star embedded in OMC1, the highly obscured radio-source, Source I, located $\sim 10^{\prime \prime}$ southeast of BN (Menten $\&$ Reid 1995), is moving toward the southeast in a direction nearly opposite to the motion of BN. The proper motion of Source I has been measured to be $14.4 \mathrm{~km} \mathrm{~s}^{-1}$ toward PA = $142^{\circ}$ (Gómez et al. 2008), $11.5 \mathrm{~km} \mathrm{~s}^{-1}$ toward PA $=109^{\circ}$ (Goddi et al. 2011b), and $9.6 \mathrm{~km} \mathrm{~s}^{-1}$ toward PA $=159^{\circ}$ (Dzib et al. 2016). Source I has a blueshifted radial velocity of approximately $-4 \mathrm{~km} \mathrm{~s}^{-1}$ with respect to $\mathrm{OMC1}$, and is thus moving in a direction roughly opposite to BN (Plambeck et al. 2009; Plambeck \& Wright 2016).

The trajectories of BN and radio Source I intersected within a 1" ( 400 au) diameter region between their current locations about 500 years ago, indicating that they may have been ejected from there (Gómez et al. 2008; Goddi et al. 2011b). If this model is correct, momentum conservation implies that Source I must have a mass of $\sim 22 \pm 3 M_{\odot}$ (Goddi et al. 2011b). However, mass estimates based on the rotation curve of the Source I disk suggest a considerably lower mass 
between 5 and $7 M_{\odot}$ (Matthews et al. 2010; Plambeck et al. 2013; Plambeck \& Wright 2016).

Another star known as infrared Source $\mathrm{n}$ (Lonsdale et al. 1982), located a few arcseconds southwest of Source I, was also found to be moving with a proper motion of $\sim 26 \mathrm{~km} \mathrm{~s}^{-1}$ toward $\mathrm{PA}=180^{\circ}$ away from the region from which Source I and BN were ejected (Gómez et al. 2008). However, Goddi et al. (2011b) failed to detect any motion. Recently, the radio proper motion of Source $\mathrm{n}$ was re-measured and found to have a value of $\sim 15 \mathrm{~km} \mathrm{~s}^{-1}$ toward PA $=179^{\circ}$ (Dzib et al. 2016). Source $\mathrm{n}$, which in the radio is a $\sim 0$ ". 4 separation double source, was found to have ejected a onesided radio jet around 2006, which has affected previous determinations of its proper motion (Dzib et al. 2016). The luminosity of Source $\mathrm{n}$ is poorly constrained to be around 2000 $L_{\odot}$ implying a mass of around 3-6 $M_{\odot}$ (Greenhill et al. 2004). The errors on the most recent proper motion measurements of BN, Source I, and $\mathrm{n}$ by Dzib et al. (2016) are about $2 \mathrm{~km} \mathrm{~s}^{-1}$ and $\pm 1^{\circ}$ to $2^{\circ}$ in total velocity on the plane of the sky and in position angle. For Source I and BN, the variations in the measured proper motions mostly reflect different choices of the reference frame in which the motions are measured. Thus, the OMC1 cloud core appears to have experienced a dynamical decay of a system of massive stars.

Infrared source IRc4, located $\sim 6^{\prime \prime}$ southwest of Source I was also found to be a $\sim 2 \times 10^{4} L_{\odot}$ protostar (De Buizer et al. 2012). Thus, in addition to the ejected sources, there may be additional massive protostars embedded in OMC1. Radio Source I, the BN object, and IRc4 are the most luminous objects in OMC1, which has a luminosity of $\sim 10^{5} L_{\odot}$.

There are two models for the ejection of runaway stars (Gies $\&$ Bolton 1986). When the most massive member of a binary explodes as a supernova, the orbital motion of the companion can be converted into linear motion (Tauris \& Takens 1998; Hoogerwerf et al. 2000, 2001; Dray et al. 2005). Alternatively, ejections can occur by $\mathrm{N}$-body interactions in dense clusters, interactions between single stars and binaries, binaries and binaries, or the dynamical decay of non-hierarchical groups (Gualandris et al. 2004; Gvaramadze \& Gualandris 2011).

The acceleration of young runaway stars requires the dynamical interaction of at least three stars, which leads to the formation or hardening of a compact binary whose gravitational binding energy powers the ejection (Reipurth et al. 2010; Reipurth \& Mikkola 2012, 2015). Goddi et al. (2011b) and Moeckel \& Goddi (2012) assumed that Source I is a compact binary, which along with $\mathrm{BN}$ was ejected from OMC1. Their numerical simulations of three-body interactions show that the most likely initial configuration is a pre-existing binary containing a massive star and $\mathrm{BN}$ that experienced a close encounter with a massive star in which the single star exchanged places with $\mathrm{BN}$ and ejected the latter. The resulting binary must have a much smaller semimajor axis than the initial binary. The recent measurement of the proper-motion of Source n (Dzib et al. 2016) suggests that a fourth star was involved. If source $\mathrm{n}$ is a wide binary, as indicated by the radio data, then at least five stars were involved in the dynamical interaction.

\subsection{The OMC1 Outflow}

OMC1 contains a wide opening-angle, arcminute-scale outflow traced by millimeter and sub-millimeter emission lines that exhibit broad $\left(>100 \mathrm{~km} \mathrm{~s}^{-1}\right)$ wings in molecules such as $\mathrm{CO}$, SO, and $\mathrm{SiO}$ (Snell et al. 1984), high-velocity $\mathrm{H}_{2} \mathrm{O}$ and
SiO maser emission (Genzel et al. 1981; Greenhill et al. 1998; Matthews et al. 2010), and hundreds of individual bow shocks in the near-IR lines of $\mathrm{H}_{2}$ and [Fe II] dubbed the "Orion fingers" (Allen \& Burton 1993; Colgan et al. 2007). Over a dozen shocks protrude into the Orion Nebula as Herbig-Haro (HH) objects (Axon \& Taylor 1984; O'dell et al. 1997a, 1997b; Kaifu et al. 2000; Reipurth \& Bally 2001; Doi et al. 2002; Graham et al. 2003; Doi et al. 2004) demonstrating that the outflow is located within $\sim 0.1 \mathrm{pc}$ of the ionization front at the rear of the Nebula. The HH objects and [Fe II] knots north and northwest of $\mathrm{OMC} 1$, located at the largest projected distance from this core, exhibit proper motions of over $400 \mathrm{~km} \mathrm{~s}^{-1}$, indicating an $\sim 500$ year age of the outflow, similar to the time since the ejection of the stars (Jones \& Walker 1985; Lee \& Burton 2000; Doi et al. 2002; Bally et al. 2011, 2015).

Observations of molecules show that the OMC1 outflow contains at least $8 M_{\odot}$ of accelerated gas in a roughly $0.2 \mathrm{pc}$ $\left(6 \times 10^{17} \mathrm{~cm}\right)$ diameter region (Snell et al. 1984). Half of the mass has an expansion velocity below $20 \mathrm{~km} \mathrm{~s}^{-1}$ with respect to $\mathrm{OMC1}$; the rest is traced by fading red- and blueshifted line-wings extending to over $100 \mathrm{~km} \mathrm{~s}^{-1}$ with respect to the radial velocity of the emission from the Orion A cloud. The outflow exhibits a slight elongation with redshifted emission to the southeast and blueshifted emission toward the northwest in the lower-velocity gas. The Integral Shaped Filament that can be traced for nearly $1^{\circ}$ north-to-south behind the Orion Nebula (Johnstone \& Bally 1999) may partially block the outflow in the north-northeast and south-southwest direction (Youngblood et al. 2016).

Interferometric images of $\mathrm{CO}$ emission with a resolution of about $3^{\prime \prime}$ obtained with the SMA revealed several dozen, highvelocity streamers originating from $\mathrm{OMC} 1$ resembling a nearly isotropic explosion (Zapata et al. 2009). These streamers exhibit Hubble-law kinematics with radial velocities proportional to the projected distance from the location from which BN and Source I were ejected $\sim 500$ years ago, and point back to this location. Comparison of the $\mathrm{CO}$ with near-IR images indicates that there is a close connection between the $\mathrm{H}_{2}$ shocks and $\mathrm{CO}$ filaments.

A smaller, 8" (3300 au) long, collimated outflow traced by $\mathrm{CO}, \mathrm{SiO}$, and $\mathrm{H}_{2} \mathrm{O}$ masers emerges along a northeast-southwest axis from radio Source I, orthogonal to the arcminute-scale, lower-velocity components of the main $\mathrm{CO}$ outflow. This compact, and therefore young ( $<200$ year old) outflow emerges nearly orthogonal to the proper motion of Source I and a line connecting Source I to BN (Plambeck et al. 2009; Zapata et al. 2012). The present-day momentum and kinetic energy content of the OMC1 outflow complex is at least $160 M_{\odot} \mathrm{km} \mathrm{s}^{-1}$ and $4 \times 10^{46}$ erg to $4 \times 10^{47}$ erg (Snell et al. 1984).

We present new ALMA observations with $\sim 1^{\prime \prime}$ angular resolution covering a $2^{\prime} \times 3^{\prime}$ region containing the entire OMC1 outflow. We discuss the physical properties of the ejected gas and review a scenario that links the origin of the outflow to the dynamic ejection of massive stars from OMC1.

\section{Observations}

The OMC1 outflow was mapped with the Atacama Large Millimeter Array (ALMA) in the $1.3 \mathrm{~mm}$ atmospheric window (ALMA Band 6) using four $1.875 \mathrm{GHz}$ wide bands centered at 216, 228, 231, and $233 \mathrm{GHz}$. The spectral windows utilized a channel-width of $0.488 \mathrm{MHz}$ or an effective resolution 
(after Hanning smoothing) of about $1 \mathrm{MHz}$, providing a velocity resolution of $1.3 \mathrm{~km} \mathrm{~s}^{-1}$. These bands contain the $J=2-1{ }^{12} \mathrm{CO}$ and $\mathrm{C}^{18} \mathrm{O}$ lines, the $217.1 \mathrm{GHz}$ line of $\mathrm{SiO}$, the 219.95 GHz line of SO, and a host of other lines. Here, we only present the ${ }^{12} \mathrm{CO}$ observations. A future paper will present data on the other species present in the four $2 \mathrm{GHz}$ wide bands. The $12 \mathrm{~m}$ ALMA antennae have a $27^{\prime \prime}$ full width half maximum (FWHM) primary beam at this wavelength. The $2^{\prime} \times 3^{\prime}$ field of view containing the outflow was observed using two rectangular grids. An $80^{\prime \prime} \times 180^{\prime \prime}$ rectangular grid used 108 antenna pointings with a grid-spacing of 12 !" 9 covering the northwest part of the outflow. A $70^{\prime \prime} \times 70^{\prime \prime}$ grid using 39 pointings covered the southeast portion of the outflow. The field was re-observed with the 12-element ALMA Compact Array (ACA) of $7 \mathrm{~m}$ diameter antennas and the four $12 \mathrm{~m}$ diameter total-power antennae to provide smaller interferometer baselines and zero-spacing data.

The raw data were reduced using the Common Astronomy Software Applications package, CASA (McMullin et al. 2007). The ALMA QA2 reduction included heavy flagging to remove strong spectral lines from the continuum, as well as the standard flux, bandpass, and gain calibrations. The data presented here used the combined 12 and $7 \mathrm{~m}$ data. The $u v$ plane visibilities from the two data sets were combined using the CASA package, imaged, and CLEAN-ed using a Briggs robust weighting parameter of 0.5 , resulting in a synthesized beam with dimensions of 1 !" $36 \times 01$ !" 78 . At the $414 \mathrm{pc}$ distance of Orion (Menten et al. 2007), the linear resolution is approximately $400 \mathrm{au}$. The rms noise varies across the field because of strong extended emission from OMC1. Typical noise values in clean regions of the continuum image are 0.5 $\mathrm{mJy}$. The noise is $5 \mathrm{mJy}$ in a typical spectral line channel, though in channels with strong emission from the OMC1 outflow the r.m.s. can rise to $0.5 \mathrm{Jy}$. At the $230 \mathrm{GHz}$ frequency of the $J=2-1 \mathrm{CO}$ line, the antenna temperature scale is given by $T_{A}^{*}=20.6 \mathrm{Kelvin} / \mathrm{Jy}$ in our synthesized beam. Thus the brightness temperature sensitivity ranges from about $0.1 \mathrm{~K}$ per channel in emission-free regions to about $10 \mathrm{~K}$ per channel in the channels containing the bulk of the $\mathrm{CO}$ emission from Orion A (radial velocities from $V_{\mathrm{LSR}} \approx 8$ to $12 \mathrm{~km} \mathrm{~s}^{-1}$ ) and at high velocities within tens of arcseconds of OMC1, where the $\mathrm{CO}$ lines are bright. The reduced data cube is available in a supplementary tar.gz archive in the online version of the Journal article.

\section{Results}

The ALMA observations reveal a cluster of over 100 highvelocity $\mathrm{CO}$ streamers that trace back to a location between the BN object and Source I. Figure 1 shows the maximum flux (brightness temperature) in each pixel from $V_{\mathrm{LSR}}=-100$ to $+120 \mathrm{~km} \mathrm{~s}^{-1}$; Figure 3 shows a moment 0 image over the same radial velocity range. Figure 4 shows a color version of the maximum temperature from $V_{\mathrm{LSR}}=-100$ to $0 \mathrm{~km} \mathrm{~s}^{-1}$ in blue and from $V_{\mathrm{LSR}}=20$ to $120 \mathrm{~km} \mathrm{~s}^{-1}$ in red. The maximum brightness temperature better illustrates the relatively constant peak surface brightness along the streamers. Furthermore, in this presentation the data is only impacted by the noise in a single channel containing the brightest emission; in an integrated image, the noise increases as the square root of the number of channels in the displayed velocity interval.

The peak CO flux in the streamers is about $4.3 \mathrm{Jy} /$ beam in both the redshifted and blueshifted ranges, which corresponds to a brightness temperature of $\sim 90 \mathrm{~K}$. The streamers dominate the outflow structure at large radial velocities, are straight with lengths ranging from $5^{\prime \prime}$ to over $40^{\prime \prime}(\sim 0.08 \mathrm{pc})$, and point back to $\mathrm{J} 2000=05^{\mathrm{h}} 35^{\mathrm{m}} 14 \mathrm{~s} 34,-5^{\circ} 22^{\prime} 28$ !' 4 , within a few arcseconds of the location from which radio Source I and BN were ejected, within $1^{\prime \prime}$ of the $\mathrm{CO}$ ejection center determined by Zapata et al. (2009), and within a few arcseconds of the ejection center determined from a traceback of the $\mathrm{H}_{2}$ proper motions (Bally et al. 2011). Most streamers are barely resolved with widths ranging from $1^{\prime \prime}$ to $2^{\prime \prime}$. They exhibit low-amplitude intensity variations indicating clumpy structure and arcsecondscale wiggles. The line-widths of the streamers range from slightly under $5 \mathrm{~km} \mathrm{~s}^{-1}$ to slightly over $10 \mathrm{~km} \mathrm{~s}^{-1}$ (full width half maximum; FWHM) in a synthesized beam area. The brightest streamers, those with $T_{B}>10 \mathrm{~K}$ are confined to a $<50^{\prime \prime}$ radius circle centered at $\mathrm{J} 2000=05: 35: 14.1,-5: 22: 19$, about $10^{\prime \prime}$ north-northwest of the ejection center (Figure 2). Fading emission with $T_{B}<10 \mathrm{~K}$ can be traced farther north toward some of the $\mathrm{H}_{2}$ fingers and toward the southeast.

\subsection{High Radial-velocity Structure}

At radial velocities greater than $20 \mathrm{~km} \mathrm{~s}^{-1}$ with respect to the $V_{\mathrm{LSR}}=9 \mathrm{~km} \mathrm{~s}^{-1}$ velocity of OMC1, over $95 \%$ of the highvelocity $\mathrm{CO}$ emission originates from about 100 streamers confined to within $\sim 0.1 \mathrm{pc}$ of the ejection center. This region coincides with the brightest near-IR $\mathrm{H}_{2}$ emission from Peak 1 and Peak 2 (Youngblood et al. 2016). As shown in Figures 1-4, the streamers have an approximately spherically symmetric spatial distribution. However, there are some significant deviations from this symmetry as discussed below.

Figure 5 shows a north-south cut through the ALMA CO data cube illustrating the velocity behavior of several dozen north-south oriented streamers. The streamers exhibit largeamplitude, linear-velocity gradients, a result previously demonstrated by Zapata et al. (2009, 2011a, 2011b). An animated version of this figure illustrating the spatial-velocity behavior of the streamers at all position angles with respect to the ejection center is shown in the electronic version of this article. The streamers are mostly straight in both the images and in spatialvelocity cuts through the data cube; each consists of a stream of clumps moving along a well-defined direction with radial velocities increasing in proportion to the projected distance from the ejection center (e.g., a "Hubble flow"). The radial velocity structure of the streamers at all orientations from the dynamical ejection center shows similar behavior. The typical radial-velocity gradients range from less than 200 to over $2000 \mathrm{~km} \mathrm{~s}^{-1}$ per parsec. In some, such as the $40^{\prime \prime}$-long streamer shown in the lower right of Figure 5, the line-brightness peaks near the high-velocity end, implying either enhanced heating, or a concentration of mass at the leading, high-velocity edge. Some streamers appear brighter on their high-velocity sides. The streamers exhibit little deceleration, implying that their densities are much higher than the ambient medium through which they are moving. However, some streamers contain faint wisps of $\mathrm{CO}$ emission extending vertically in Figure 5 to lower radial velocities. They may trace gas being shorn off and decelerated as the dense clumps move supersonically through the medium.

Most streamers intersect the $6-12 \mathrm{~km} \mathrm{~s}^{-1}$ rest velocity (in $V_{\text {LSR }}$ coordinates) of the Orion A cloud within a few arcseconds of the ejection site of Source I and BN. However, extrapolating the linear velocity-gradient of the two longest 


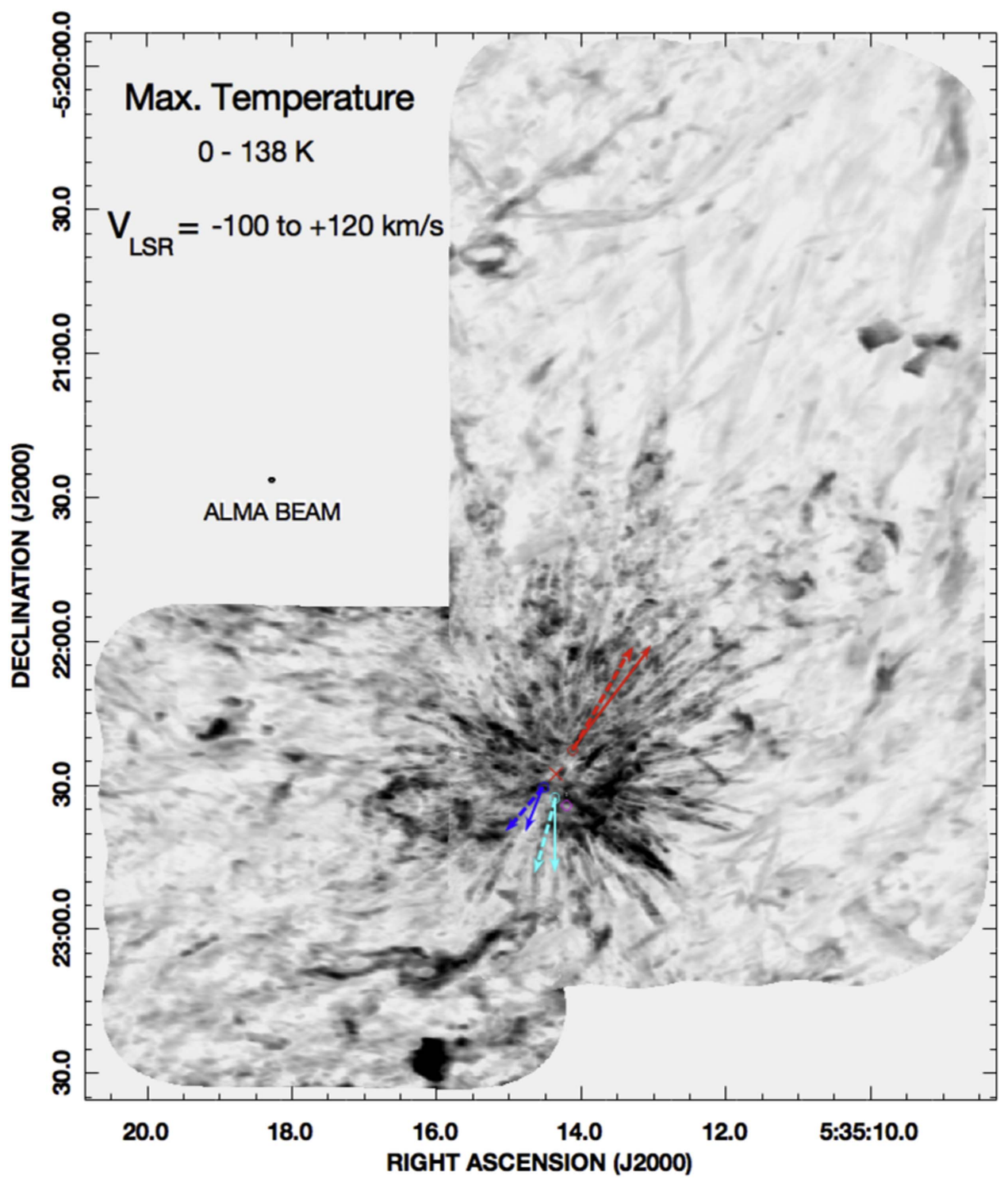

Figure 1. ALMA $1.3 \mathrm{~mm}$ image showing the high-velocity $230 \mathrm{GHz} \mathrm{CO}$ emission from the explosive OMC1 outflow with $\sim 1^{\prime \prime}(\sim 400$ au $)$ angular resolution. The maximum brightness temperatures in velocity channels covering the indicated velocity intervals are shown from $T_{A}^{*}=0$ to $89 \mathrm{~K}$ on a linear scale. The current positions of BN, Source I, Source n, and IRc4 are indicated by red, blue, cyan, and magenta circles respectively. The vectors show the proper motions of BN (red), Source I (blue), and Source n (cyan) in the Orion Nebula Cluster reference frame Dzib et al. (2016). The dashed vectors show these motions in a frame in which the vector momenta sum to zero as described in the text. The lengths of the vectors correspond to the expected proper motions in 2000 years.

streamers in Figure 5 (one in the upper left of Figure 5 and the other being the $40^{\prime \prime}$-long streamer in the lower right) back to the velocity of the $\mathrm{OMC} 1$ core and the Orion A cloud results in intersection points separated by $\sim 5^{\prime \prime}$. Fitting lines to the streamers in the spatial-velocity images also results in a spread of intersection point scattered over a roughly $5^{\prime \prime}$ diameter region. This behavior may indicate slight deflections by interactions with their environment or spreading of the ejecta. Nevertheless, the distribution of intersection points in the space-space images (Figures 1-5) peak within an arcsecond of the dynamical ejection center determined from proper motions of the stars referenced to the frame in which OMC1 is at rest, as discussed below.

Dozens of red- and blueshifted CO streamers point toward the $\mathrm{H}_{2}$ and [Fe II] bow shocks. This is particularly evident toward the southwest. Although some $\mathrm{CO}$ streamers extend right up to associated $\mathrm{H}_{2}$ bow shocks located at their highvelocity ends, others disappear between $30 \%$ to $70 \%$ of the distance to the near-IR bow shocks to which they point. Given that the $\mathrm{HH}$ object and some near-IR proper motions reach values of $\sim 400 \mathrm{~km} \mathrm{~s}^{-1}$, a factor of two to three times the highest $\mathrm{CO}$ radial velocities, it is likely that $\mathrm{CO}$ and $\mathrm{H}_{2}$ are dissociated by the intense shock heating and radiation above speeds of the order of $100-150 \mathrm{~km} \mathrm{~s}^{-1}$.

The dynamical ages of the streamers can be estimated from their maximum radial velocities if their inclination angle with respect to the line of sight, or their proper motions are known. Assuming spherical symmetry and that all streamers were produced at the same time, the maximum projected distance of the streamer-ends from the ejection center can be combined with their radial velocities to estimate their inclination angles and ages. Most bright streamers are confined to within $R_{\max }=50^{\prime \prime}\left(3.1 \times 10^{17} \mathrm{~cm}\right)$ of the explosion center. The highest radial velocities are $V_{\max }=159 \mathrm{~km} \mathrm{~s}^{-1}$ with respect to OMC1. (Knot \#1 in Table 1 has $V_{\mathrm{LSR}}=-149.5 \mathrm{~km} \mathrm{~s}^{-1}$. Since $\mathrm{OMC} 1$ is at $V_{\mathrm{LSR}}=+9 \mathrm{~km} \mathrm{~s}^{-1}$, this knot is moving with 


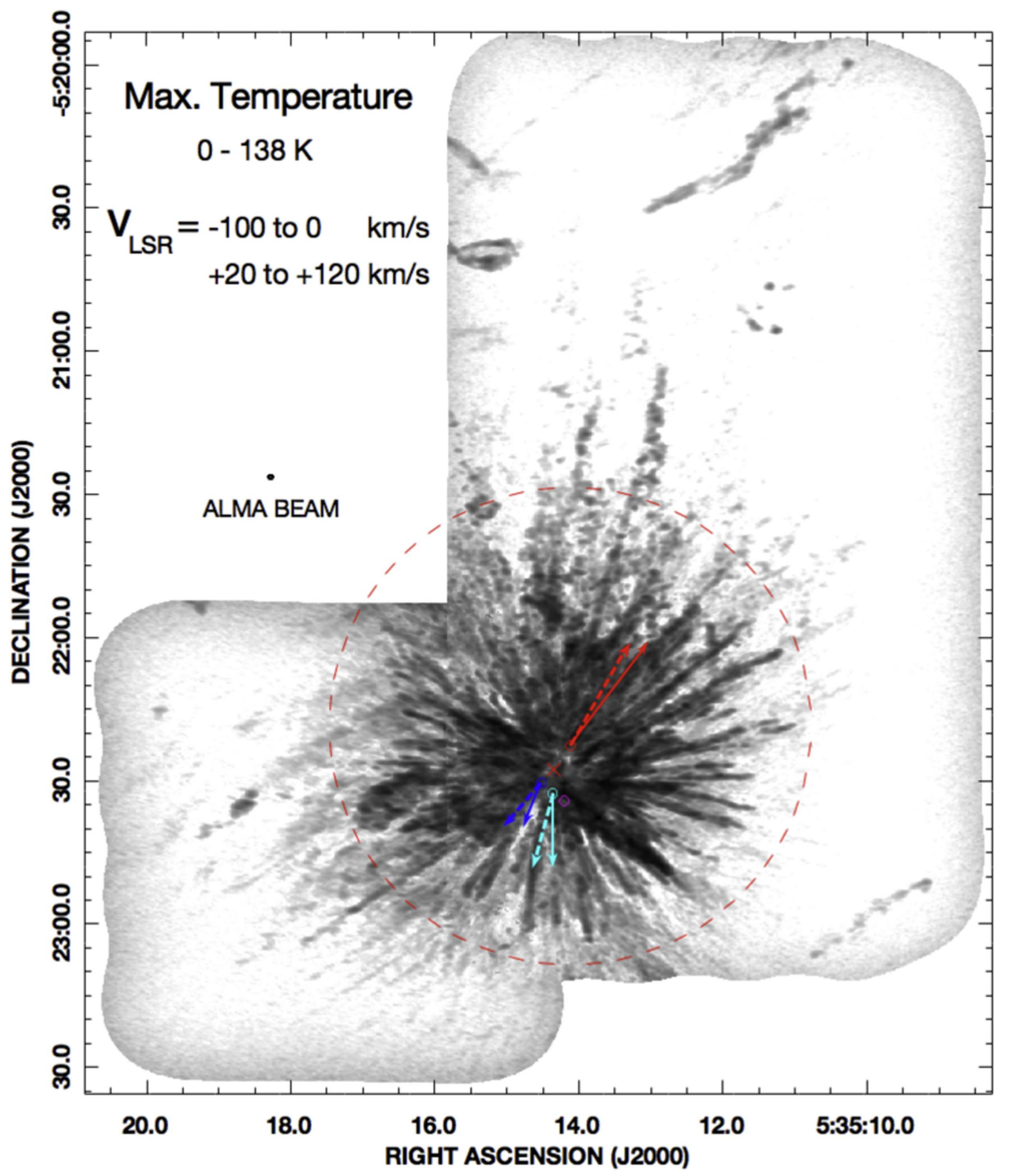

Figure 2. ALMA CO image showing only the high-velocity emission, excluding the velocity range from $\mathrm{V}_{\mathrm{LSR}}=0$ to $+20 \mathrm{~km} \mathrm{~s}^{-1}$. As in Figure 1 , the maximum brightness temperature in the indicated velocity ranges is shown. However, in this figure, the logarithm of $T_{\max }$ is shown to emphasize the low-level emission. A dashed circle with a radius of 50" enclosing most of the high-velocity CO emission is shown for reference. The center of this circle is at J2000 $=05: 35: 14.11,-5$ : 22:18.7, about $10^{\prime \prime}$ north-northwest of the ejection center. Proper motion vectors and luminous sources are shown as in Figure 1. Note the small outflows in the lower right and top of the figure. These are discussed in Section 3.6.

a velocity of at least $159 \mathrm{~km} \mathrm{~s}^{-1}$ with respect to the cloud core). If the proper motions at $50^{\prime \prime}$ projected distance were $159 \mathrm{~km} \mathrm{~s}^{-1}$, the dynamic age would be $t_{\mathrm{dyn}}=R_{\max } / V_{\max } \approx$ 620 years.

Inclination angles of individual streamers can be estimated from their maximum radial velocity, $V_{\text {radial }}$, and projected distance of their high-velocity end, $r_{\text {end }}$ from the ejection center. The ratio $r_{\text {end }} / R_{\max }$ is a measure of the inclination angle of the streamer with respect to the line of sight. Thus, $t_{\mathrm{dyn}} \approx\left(r_{\mathrm{end}} / V_{\max }\right) \tan \left[\cos ^{-1}\left(r_{\text {end }} / R_{\max }\right)\right]$. For example, the long streamer in the lower right of Figure 5 has a projected length of $r_{\text {end }} \approx 40^{\prime \prime}$ and a radial velocity with respect to OMC1, $V_{\text {radial }} \approx 110 \mathrm{~km} \mathrm{~s}^{-1}$, at its high-velocity end. For $R_{\max }=50^{\prime \prime}$, its dynamical age is $t_{\mathrm{dyn}} \approx 540$ years. The ALMA data are consistent with an age of 470 to 700 years, similar to the age obtained by Zapata et al. (2009) with the SMA and the recent analysis of the time since ejection of the high-proper motion stars from OMC1 by Dzib et al. (2016). The fastest blueshifted and redshifted knots are displaced from the ejection center in projection, indicating some deviations from spherical symmetry. Furthermore, as discussed below, many of the $\mathrm{H}_{2}$ [Fe II], and $\mathrm{HH}$ objects in the north and northwest are located up to $140^{\prime \prime}$ from the ejection center.

\subsection{Low Radial-velocity Structure}

Within $\sim 20^{\prime \prime}$ of the ejection site, the low-radial velocity CO emission within $\sim 20 \mathrm{~km} \mathrm{~s}^{-1}$ of the OMC1 rest velocity (the emission line from the core is centered at $V_{\mathrm{LSR}}$ [core] $\approx 9$ to $10 \mathrm{~km} \mathrm{~s}^{-1}$ ) becomes over-resolved in the ALMA data. The outer boundary of this region is seen as an approximately ovoidal shell with a chaotic boundary (Figure 5) with an interior region with negative intensities. The combined $12 \mathrm{~m}$ and ACA data over-resolves this region and thus there is a large amount of missing flux. Nevertheless, an abundance of small- 


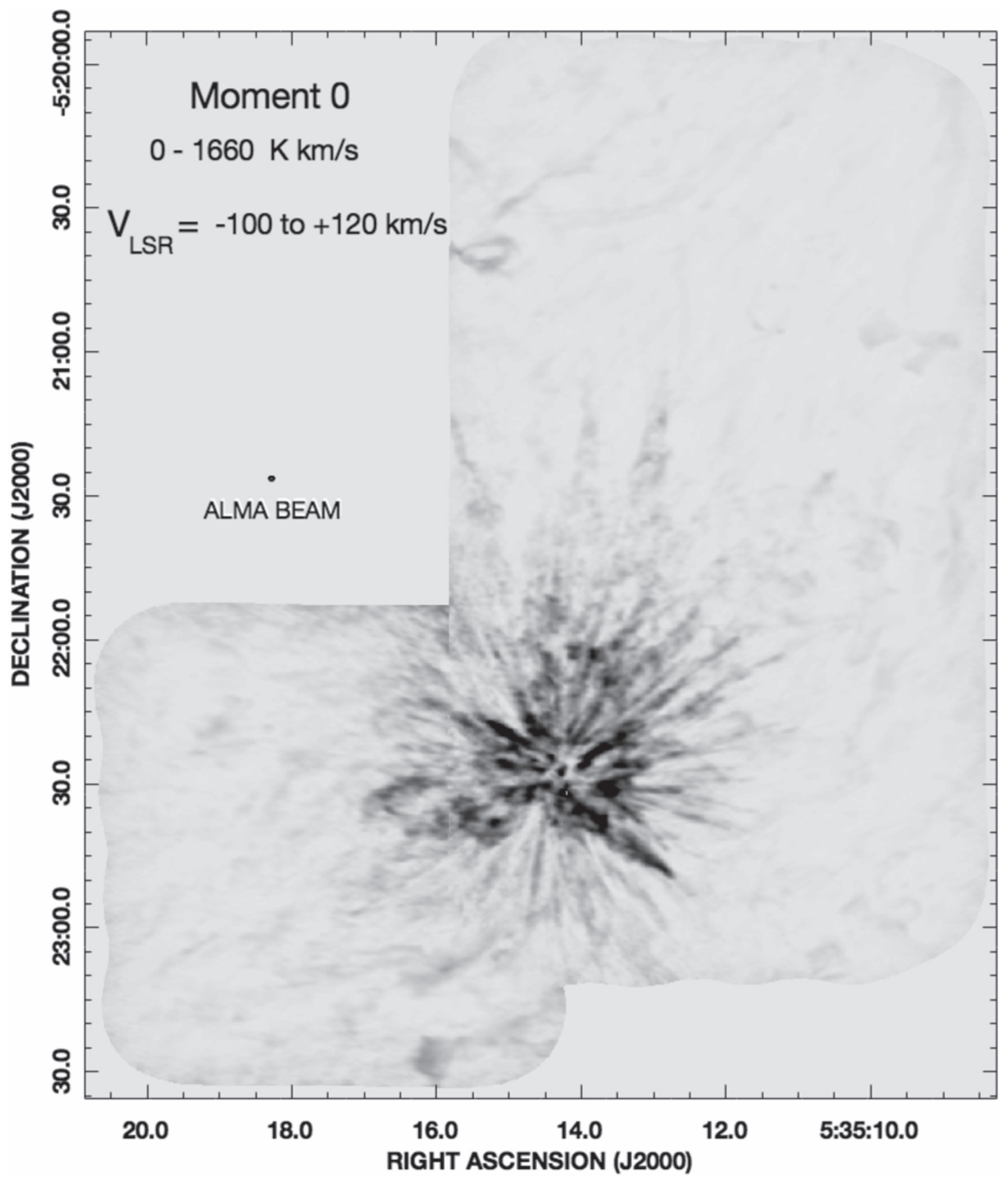

Figure 3. Moment 0 map showing the high-velocity $230 \mathrm{GHz} \mathrm{CO}$ emission from OMC.

scale structure can be seen at low radial velocities and beyond $20^{\prime \prime}$ of the core.

Within $\sim 10 \mathrm{~km} \mathrm{~s}^{-1}$ of the rest velocity of OMC1, the outflow is elongated from southeast to northwest with a length of nearly $180^{\prime \prime}$ ( $0.36 \mathrm{pc}$ : Figure 6$)$ and contains several shelllike structures. An arcminute long, 30" wide, blueshifted bubble with walls several arcseconds-wide $(\sim 1000 \mathrm{au})$ extends southeast at low blueshifted radial velocities of $V_{\mathrm{LSR}} \sim 0$ to $7 \mathrm{~km} \mathrm{~s}^{-1}$; its southern rim is located behind the Trapezium stars. A slightly smaller, redshifted bubble protrudes due east of the OMC1 cloud core at $V_{\mathrm{LSR}} \sim 12$ to $\sim 55 \mathrm{~km} \mathrm{~s}^{-1}$. A broad fan of low-velocity red and blueshifted ejecta extends about 1.5 toward the northwest, in a direction opposite to the southeast oriented blueshifted bubble. These bubbles are marked in Figure 6.

Several limb-brightened "fingers" of $\mathrm{CO}$ emission extend north and northwest for $60^{\prime \prime}-130^{\prime \prime}$ at low-radial velocities. CO emission is clearly associated with the $\mathrm{H}_{2}$ fingers \#2, 3, 5, and 8 (Figure 7). Both redshifted and blueshifted $\mathrm{CO}$ with velocities of \pm 3 to $20 \mathrm{~km} \mathrm{~s}^{-1}$ with respect to $V_{\mathrm{LSR}}=9 \mathrm{~km} \mathrm{~s}^{-1}$ are associated with the $\mathrm{H}_{2}$ fingers $\# 2$ and \#5, suggesting that they lie close to the plane of the sky. Low-radial velocity material in fingers \#3 and \#5 is located in the interior of the $\mathrm{H}_{2}$ wakes and likely traces molecules moving at high velocities nearly in the plane of the sky (Figure 7). The elongation of the low-velocity bubbles and the fingers are roughly parallel to the motions of BN and Source I.

Figure 8 shows the integrated $\mathrm{CO}$ emission at the core radial velocities, $V_{\mathrm{LSR}}=6.7-12.6 \mathrm{~km} \mathrm{~s}^{-1}$. Conical ridges of enhanced $\mathrm{CO}$ emission best seen in Figure 8 are located just outside $\mathrm{H}_{2}$ fingers 3 and 5 and may trace limb-brightened ambient gas that has been displaced and slightly accelerated to redshifted velocities by the passage of high-velocity ejecta. At the core velocities, the $\mathrm{CO}$ emission from fingers \#2 and \#5 consists of a pair of converging filaments located just outside the associated near-IR $\mathrm{H}_{2}$ emission. These "upside-down V-shaped" features may trace very low-velocity CO swept-up from the ambient medium.

Figure 9 shows a high-resolution image of the outflow from Bally et al. (2015). Figure 10 shows a cartoon illustrating the various features discussed above. 


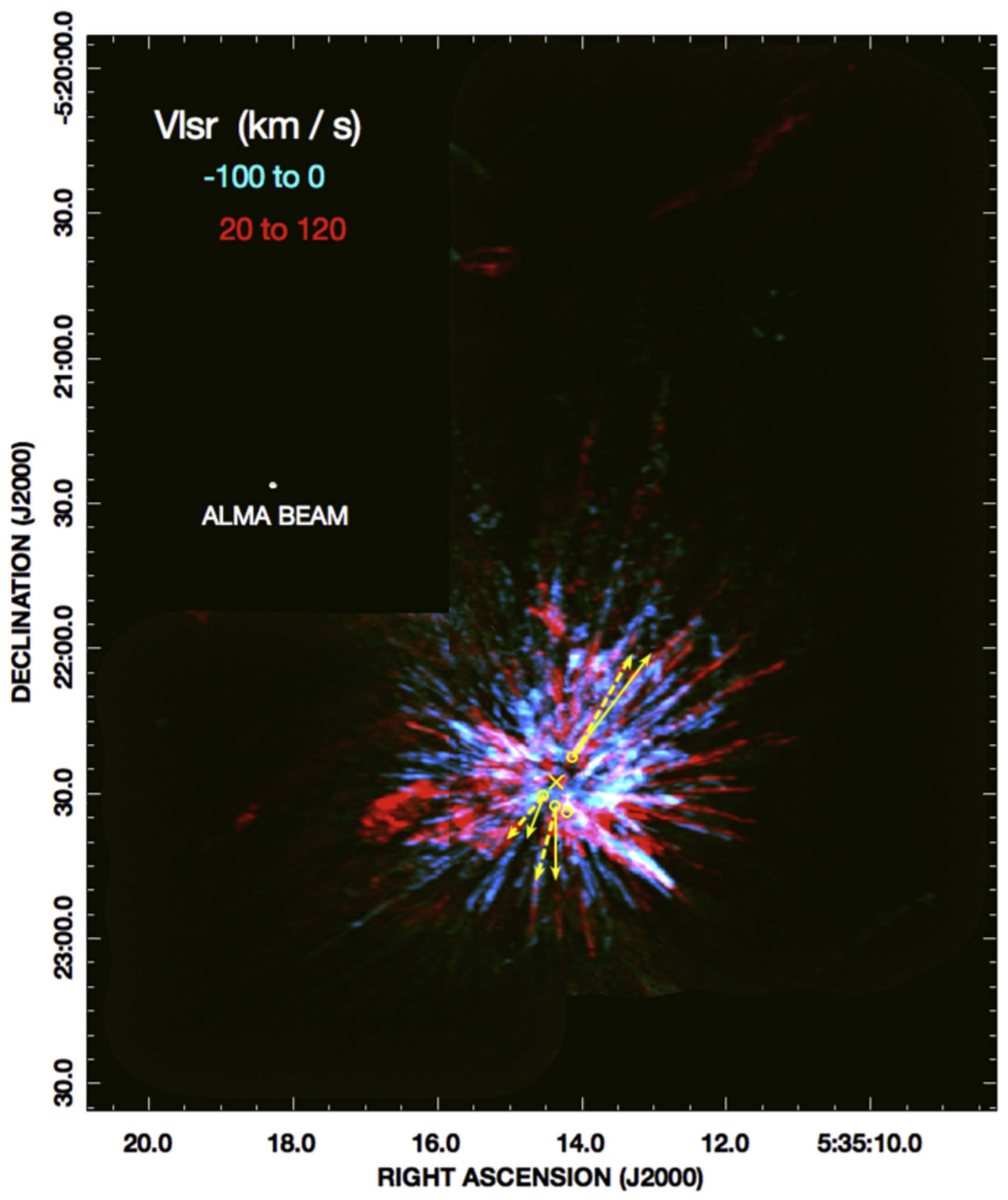

Figure 4. Blue-green features show the outflow emission from $V_{\mathrm{LSR}}=-100$ to $0 \mathrm{~km} \mathrm{~s}^{-1}$; red features show emission from $V_{\mathrm{LSR}}=+20$ to $+120 \mathrm{~km} \mathrm{~s}$. The yellow circles show the locations of the massive stars ejected from OMC1; from top to bottom these are the BN object, Source I, Source n, and IRc4. The yellow cross marks the location of the explosion center measured from the proper motions of near-infrared $\mathrm{H}_{2}$ emission features (Bally et al. 2011); this location coincides with the explosion center determined from the orientations of the CO streamers to within a few arcseconds (Zapata et al. 2009). Several unrelated CO outflows from stars outside the OMC1 core can be seen near the top and lower right corner of this image.

\subsection{Deviations from Spherical-symmetry}

The redshifted emission is dominated by the low-radialvelocity bubble pointing due-east of the OMC1 core and a spray of several dozen high-velocity streamers oriented toward the west. The reshifted bubble has an axis of symmetry pointing toward $\mathrm{PA} \approx 95^{\circ}$ from $\mathrm{OMC} 1$. Except for the young and bright Source I outflow and a single redshifted streamer at $\mathrm{PA} \approx 70^{\circ}$, there are no redshifted streamers between $\mathrm{PA}=25^{\circ}$ and $170^{\circ}$. In contrast, the blueshifted streamers exhibit a more isotropic distribution.

The largest observed radial velocities in $\mathrm{CO}$ are at $V_{\mathrm{LSR}}=-142 \mathrm{~km} \mathrm{~s}^{-1} 58^{\prime \prime}$ north of the ejection center, and $+133 \mathrm{~km} \mathrm{~s}^{-1}$ about $74^{\prime \prime}$ north, significantly outside the $50^{\prime \prime}$ radius region containing most of the bright, high-velocity $\mathrm{CO}$ streamers. Table 1 lists some of the highest velocity streamer ends; their locations are marked in Figure 7. The most prominent $\mathrm{H}_{2}$ and [Fe II] fingers are marked with red lines in
Figure 7, where they are numbered 1 through 8 . The propermotions of several of these fingertips reach $V \sim 400 \mathrm{~km} \mathrm{~s}^{-1}$ in $\mathrm{H}_{2}$ and [Fe II], nearly three times faster than the highest radialvelocity $\mathrm{CO}$ streamers.

The low radial-velocity $\mathrm{CO}$ emission extends outside a $50^{\prime \prime}$ radius circle containing most streamers. Additionally, streams of ejecta extend to the north and northwest, where the highest proper motion near-infrared "fingers" of $\mathrm{H}_{2}$ and "fingertips" of [Fe II] are found. As discussed below, both red- and blueshifted low-velocity $\mathrm{CO}$ is associated with the bases of several of these fingers (Figure 6), indicating that there is an unusually fast fan of ejecta moving close to the plane of the sky toward the north and northwest.

Models of binary in-spiral indicate ejection of debris along the orbital plane (Soker \& Tylenda 2006). Given the proper motions of the BN object and Source I, the most likely final encounter direction is along a southeast-northwest axis. Ejecta 


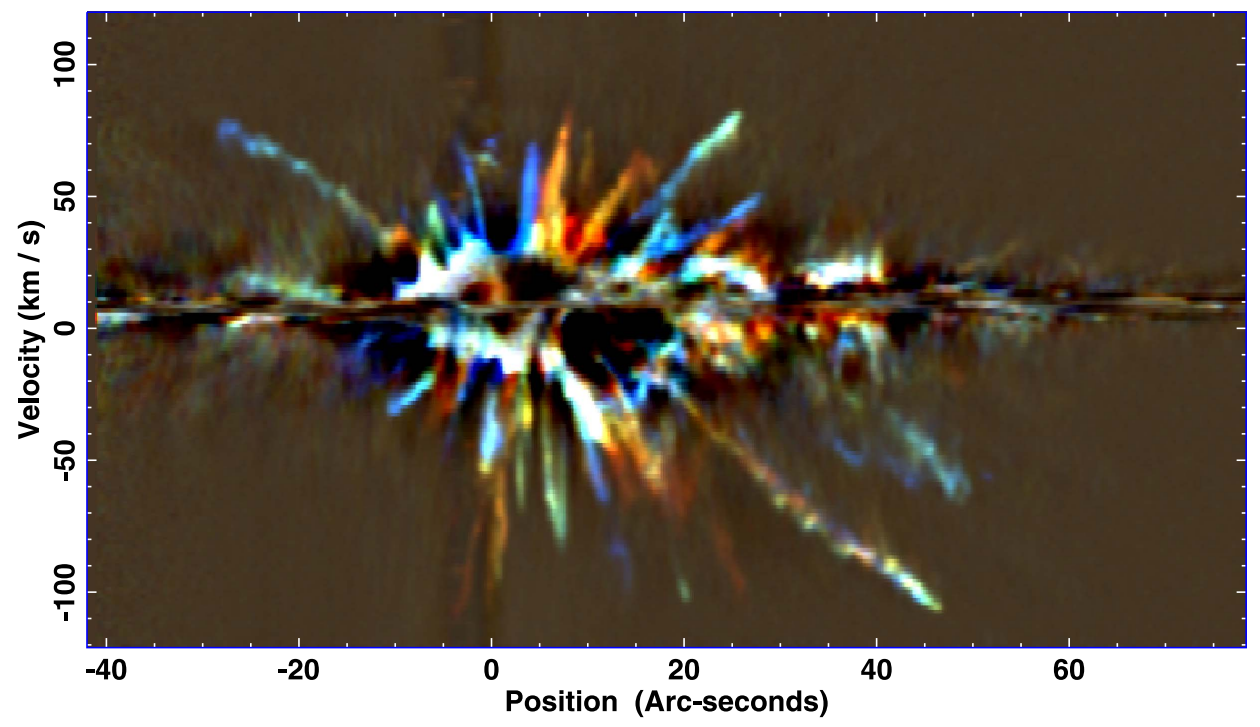

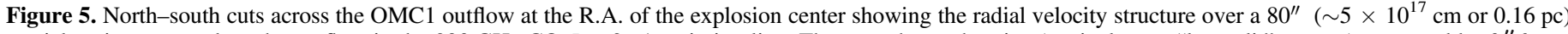

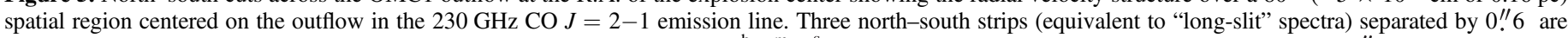

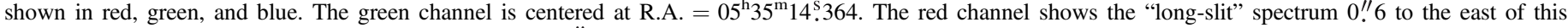

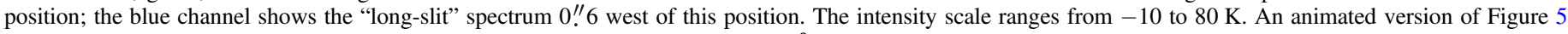

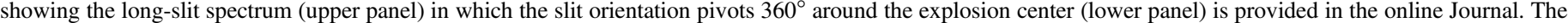
animation is $15 \mathrm{~s}$ in duration.

(An animation of this figure is available.)

Table 1

Highest Radial Velocity CO Knots in Orion OMC1

\begin{tabular}{lccrrl}
\hline \hline No. & $\begin{array}{c}\alpha(\mathrm{J} 2000)(1) \\
\left(05^{\mathrm{h}} 35^{\mathrm{s}}\right)\end{array}$ & $\begin{array}{c}\delta(\mathrm{J} 2000)(2) \\
\left(-05^{\circ}\right)\end{array}$ & $\begin{array}{c}V_{\mathrm{LSR}}(3) \\
\left(\mathrm{km} \mathrm{s}^{-1}\right)\end{array}$ & $\begin{array}{r}D(4) \\
\left({ }^{\prime \prime}\right)\end{array}$ & Direction \\
\hline 1 & 13.99 & $-22: 11.9$ & -149.5 & 17.8 & $\mathrm{~N}$ \\
2 & 14.76 & $-22: 31.6$ & -148.0 & 6.4 & $\mathrm{E}$ \\
3 & 13.00 & $-22: 15.8$ & -140.7 & 24.0 & $\mathrm{NW}$ \\
4 & 14.07 & $-22: 06.7$ & -140.7 & 22.6 & $\mathrm{~N}$ \\
5 & 14.32 & $-21: 30.7$ & -137.8 & 58.0 & $\mathrm{~N}$ \\
6 & 13.81 & $-21: 56.6$ & -136.3 & 33.2 & $\mathrm{NNW}$ \\
7 & 14.50 & $-22: 36.9$ & -130.5 & 8.5 & $\mathrm{~S}$ \\
8 & 15.05 & $-22: 20.7$ & -130.5 & 13.1 & $\mathrm{NE}$ \\
9 & 13.58 & $-21: 15.4$ & 145.4 & 74.3 & $\mathrm{~N}$ \\
10 & 13.63 & $-21: 17.5$ & 142.0 & 72.0 & $\mathrm{~N}$ \\
11 & 14.10 & $-22: 36.9$ & 145.0 & 9.0 & $\mathrm{SW}$ \\
15 & 12.98 & $-22: 08.4$ & 129.4 & 29.0 & $\mathrm{NW}$ \\
16 & 13.67 & $-21: 24.7$ & 129.4 & 64.8 & $\mathrm{~N}$ \\
12 & 14.86 & $-22: 26.3$ & 129.4 & 8.0 & $\mathrm{NE}$ \\
13 & 14.66 & $-22: 27.8$ & 129.4 & 4.7 & $\mathrm{NE}$ \\
14 & 16.32 & $-22: 37.1$ & 129.4 & 30.5 & SEE \\
17 & 12.50 & $-21: 58.8$ & 123.5 & 40.8 & $\mathrm{NW}$ \\
18 & 12.66 & $-22: 34.9$ & 123.5 & 26.1 & SWW \\
\hline
\end{tabular}

Note. (1) Seconds of R.A. (2) Arcminutes and arcseconds of decl. (3) $V_{\text {LSR }}$ is the radial velocity in the Local Standard of Rest frame in which the OMC1 has $V_{\mathrm{LSR}}=9 \mathrm{~km} \mathrm{~s}^{-1}$. (4) Projected distance in arcseconds from the suspected ejection center located at $\mathrm{J}(2000)=05^{\mathrm{h}} 35^{\mathrm{m}} 14^{\mathrm{s}} .34,-5^{\circ} 22^{\prime} 28^{\prime \prime}$. 4. (5) Direction from $\mathrm{OMC} 1$.

launched during the in-spiral phase are expected to be launched preferentially in this direction. The observed low radialvelocity outflow and the fan of high-proper-motion ejecta is elongated along the axis defined by the stellar proper motion vectors, consistent with the ejection of material in the encounter plane. These asymmetric features are marked in the cartoon shown in Figure 10.
As discussed above, a 30" wide by $70^{\prime \prime}$ long bubble of limbbrightened, blueshifted emission extends toward the southeast with an axis of symmetry at PA $\sim 130^{\circ}-140^{\circ}$. Figure 6 shows that at low radial velocities, there is a wide-angle fan of both red- and blueshifted debris extending to about $90^{\prime \prime}$ from OMC1 at $\mathrm{PA} \sim 310^{\circ}$, roughly opposite of the blueshifted bubble.

The brightest $\mathrm{CO}$ emission away from the $\mathrm{OMC} 1$ rest velocity is associated with the compact, 200 to 300 year old, northeastsouthwest ejecta from Source I (Zapata et al. 2012). Although this component is difficult to separate from the other streamers in the $1.3 \mathrm{~mm} \mathrm{CO}$ data cubes, it is very apparent in a number of other molecules such as $\mathrm{SiO}$ and even $\mathrm{C}^{18} \mathrm{O}$. Thus, Source I continues to power a very dense bipolar outflow roughly orthogonal to its proper motion.

\subsection{Column Densities and Masses}

Far-infrared observations of high rotational states of $\mathrm{CO}$ with the Herschel space telescope (Goicoechea et al. 2015) show that the spectral line energy distribution (SLED) can be well fit with three components having temperatures of 200, 500, and $2500 \mathrm{~K}$. The ALMA data cube shows that most streamers have brightness temperatures between 20 and $90 \mathrm{~K}$ and Doppler widths of $\Delta V \sim 5$ to $10 \mathrm{~km} \mathrm{~s}^{-1}$. Assuming cylindrical geometry, a characteristic width or depth of 1 !'2 (500 au), and a ${ }^{12} \mathrm{CO}$ abundance $X_{\mathrm{CO}}=10^{-4} n\left(\mathrm{H}_{2}\right)$, the relation between column density of $\mathrm{CO}$ and the density of $\mathrm{H}_{2}$ is $N_{\mathrm{CO}}=7.5 \times 10^{11} n\left(\mathrm{H}_{2}\right) \mathrm{cm}^{-2}$.

The column density and density of the streamers depends on the assumed gas and radiation temperatures. If the $\mathrm{CO}$ emission we observe originates in the $200 \mathrm{~K}$ component and the background radiation temperature is $10 \mathrm{~K}$, appropriate for the outer parts of the OMC1 outflow, the radiative transfer and excitation code RADEX (van der Tak et al. 2007) indicates that the $\mathrm{CO}$ excitation temperature in the $J=2-1$ line ranges from 25 to $88 \mathrm{~K}$ for $\mathrm{H}_{2}$ densities $n\left(\mathrm{H}_{2}\right)=4 \times 10^{5}$ to $2 \times 10^{6} \mathrm{~cm}^{-3}$ for $\Delta V \sim 5 \mathrm{~km} \mathrm{~s}^{-1}$ wide lines. For a background radiation 


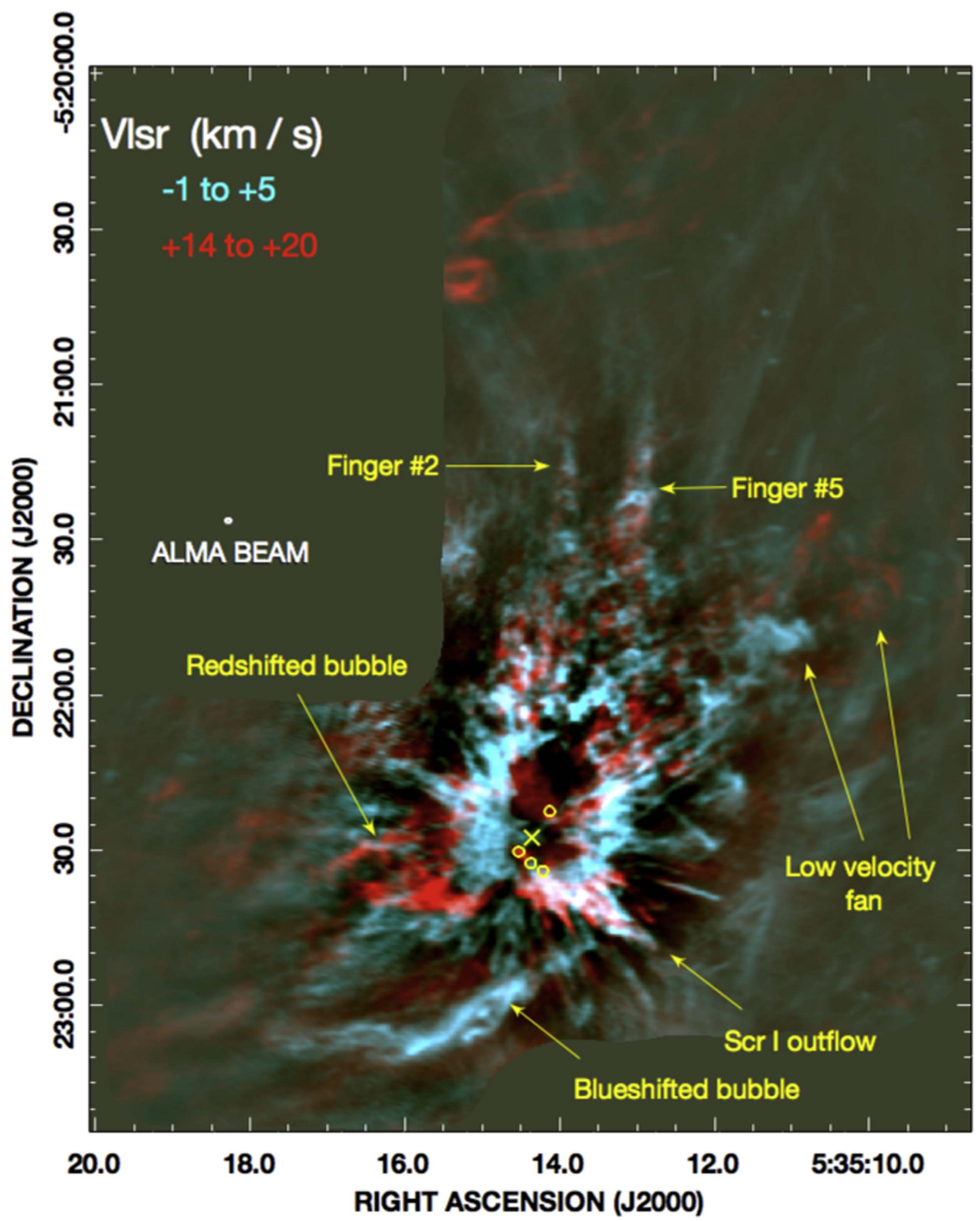

Figure 6. ALMA $1.3 \mathrm{~mm}$ image showing the $230 \mathrm{GHz} \mathrm{CO} J=2-1$ emission at low radial-velocities formed by integrating the line intensity over a velocity range from $V_{\mathrm{LSR}}=-1$ to $+5 \mathrm{~km} \mathrm{~s}^{-1}$ (shown in cyan) and $V_{\mathrm{LSR}}=+14$ to $+20 \mathrm{~km} \mathrm{~s}^{-1}$ (shown in red). The ejected massive stars and outflow ejection center are shown as in Figure 4. Several additional outflows from young stars in the Orion A cloud can also be seen in this velocity range toward the north side of the image. The displayed intensities range from -7 to $63 \mathrm{~K}$.

temperature of $100 \mathrm{~K}$, appropriate for regions close to (within a few arcseconds) the massive stars, the observed $\mathrm{CO}$ brightness requires densities of $10^{6}-10^{7} \mathrm{~cm}^{-3}$. The $\mathrm{CO}$ opacities range from 0.3 to 3 for both cases. These optical depths are consistent with the non-detection of $\mathrm{C}^{18} \mathrm{O}$ at a brightness temperature above $0.2 \mathrm{~K}(10 \mathrm{mJy} /$ beam $)$ at high radial velocities. Given the peak brightness temperature in the streamers of $\sim 90 \mathrm{~K}$, the lowest allowed kinetic temperature is $100 \mathrm{~K}$. In this case, the acceptable volume density ranges from $\sim 10^{6}$ to $\sim 10^{7} \mathrm{~cm}^{-3}$ for a background radiation temperature of $10 \mathrm{~K}$. For line-widths of $10 \mathrm{~km} \mathrm{~s}^{-1}$, the line opacities are approximately a factor of two lower and the densities required to produce the observed line brightness are about a factor of two higher than for the estimates for $5 \mathrm{~km} \mathrm{~s}^{-1}$ wide lines. It is likely that there is a wide range of gas kinetic temperatures in each streamer with hot $\sim 10^{3} \mathrm{~K}$ gas near their leading edges and sides where shock radiation and compression heat the post-shock gas to below $200 \mathrm{~K}$ in their interiors where radiative cooling dominates. Lowering the $\mathrm{CO}$ abundance increases the required density.

Column densities can also be estimated using the $\mathrm{X}$-factor method (Bolatto et al. 2013), which gives $N\left(\mathrm{H}_{2}\right) \approx$ $2.6 \times 10^{20} T_{A}^{*} \Delta V$, where $T_{A}^{*}$ is the antenna temperature corrected for atmospheric attenuation and $\Delta V$ is the effective width of the $\mathrm{CO}$ spectra. The conversion between the $\mathrm{CO}$ flux in Jy per beam and mass of hydrogen in the beam is $M\left(\mathrm{H}_{2}\right) \approx 1.2 \times 10^{30} S_{\text {beam }}(\mathrm{Jy}) \mathrm{g} \quad\left(\sim 6 \times 10^{-4} S_{\text {beam }}(\mathrm{Jy}) M_{\odot}\right)$, where $S_{\text {beam }}$ (Jy) is flux density in a $1.46 \mathrm{~km} \mathrm{~s}^{-1}$-wide channel. The mass contained in each $\sim 1^{\prime \prime}$ resolution element ranges from $\sim 10^{29}$ to slightly over $3 \times 10^{30} \mathrm{~g}$. Summing over the spatial and velocity extents of the filaments gives masses ranging from a few times $10^{29} \mathrm{~g}$ for faint features only a few 


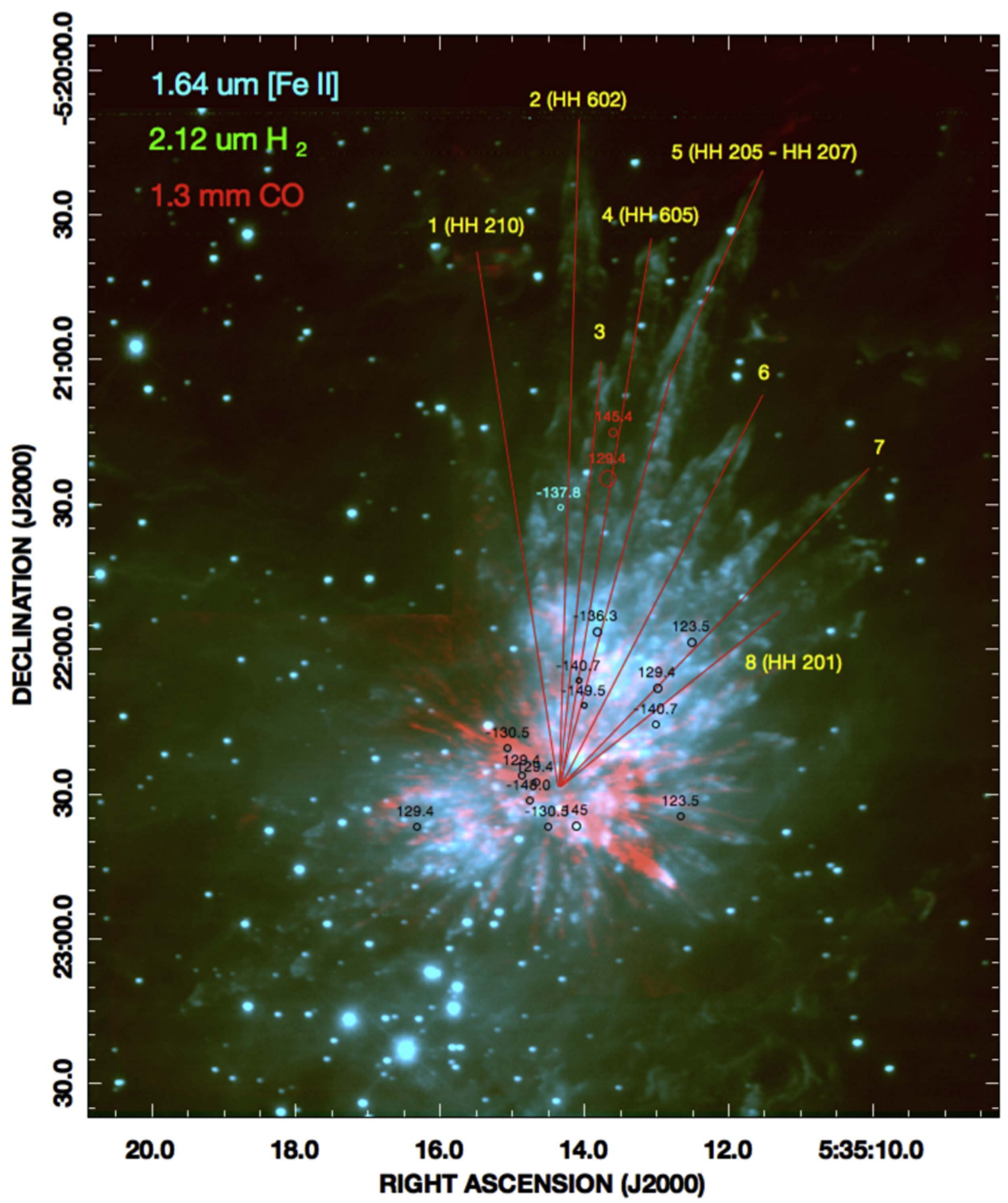

Figure 7. Maximum brightness temperature CO maps in the redshifted and blueshifted radial velocity ranges shown in Figure 1 covering the high-velocity emission from -100 to $+120 \mathrm{~km} \mathrm{~s}^{-1}$ (red) shown superimposed on the $2.12 \mu \mathrm{m}$ fingers of shock-excited $\mathrm{H}_{2}$ emission (green) from the OMC1 outflow (Bally et al. 2011). The massive Trapezium stars responsible for ionization of the Orion Nebula appear near the bottom of this image. The eight most prominent $\mathrm{H}_{2}$ fingers are marked with red lines. The $\mathrm{HH}$ objects located at their ends are indicated. The small red and black circles indicate the locations and radial velocities of the highest velocity streamer ends listed in Table 1.

arcseconds long to over $10^{32} \mathrm{~g}$ for the longest streamers. The total mass of the streamers traced by $\mathrm{CO}$ having a radial velocity that differs from the ambient cloud by more than $20 \mathrm{~km} \mathrm{~s}^{-1}$ is $M_{\text {fast }} \sim 1 M_{\odot}$.

Integration of the $\mathrm{CO}$ data cube over an $86^{\prime \prime}$ diameter circle and summing the flux over the radial velocity ranges -100 to $0 \mathrm{~km} \mathrm{~s}^{-1}$ and +20 to $120 \mathrm{~km} \mathrm{~s}^{-1}$ implies a mass of $1.0 \mathrm{M}_{\odot}$ using this method. The spatial-filtering of the interferometer severely limits our ability to estimate masses within the inner $\pm 10 \mathrm{~km} \mathrm{~s}^{-1}$ of the outflow; even at higher velocities, extended emission is filtered out and thus our mass estimates are lower bounds.

\subsection{The Relationship of the $\mathrm{CO}$ Streamers and the $\mathrm{H}_{2}$ Fingers}

The $\mathrm{H}_{2}$ fingers and [Fe II] bow shocks extend $\sim 140^{\prime \prime}$ from the ejection center toward the north and northwest (Figure 9). The multi-transition analysis of the $\mathrm{H}_{2}$ in the OMC1 outflow
(Youngblood et al. 2016) shows that near the core, the $\mathrm{H}_{2}$ emission is primarily blueshifted while toward the outer parts toward the north, northwest, and the southeast, the $\mathrm{H}_{2}$ emission is both red- and blueshifted. Oh et al. (2016) present high spectral resolution data cubes of $\mathrm{H}_{2}$ toward a $15^{\prime \prime} \times 13^{\prime \prime}$ field centered on the brightest portion of the Orion outflow $\sim 25^{\prime \prime}$ north-northwest of the ejection center. The $\mathrm{H}_{2}$ emission in this field has morphology and kinematics similar to the $\mathrm{CO}$ streamers in the region with red- and blueshifted emission extending from -105 to $+95 \mathrm{~km} \mathrm{~s}^{-1}$.

The largest CO Doppler shifts, -150 and $+145 \mathrm{~km} \mathrm{~s}^{-1}$ are located within $\sim 60^{\prime \prime}$ of the ejection center (Table 1 and Figure 5). These line-of-sight motions are about a factor of two smaller than the highest proper motions in $\mathrm{H}_{2},[\mathrm{Fe} \mathrm{II}]$, and associated $\mathrm{HH}$ objects, $200-400 \mathrm{~km} \mathrm{~s}^{-1}$ (Doi et al. 2002) located $100^{\prime \prime}-140^{\prime \prime}$ north and northwest of the ejection center (see Figures 11 through 17). The terminal radial velocities of 


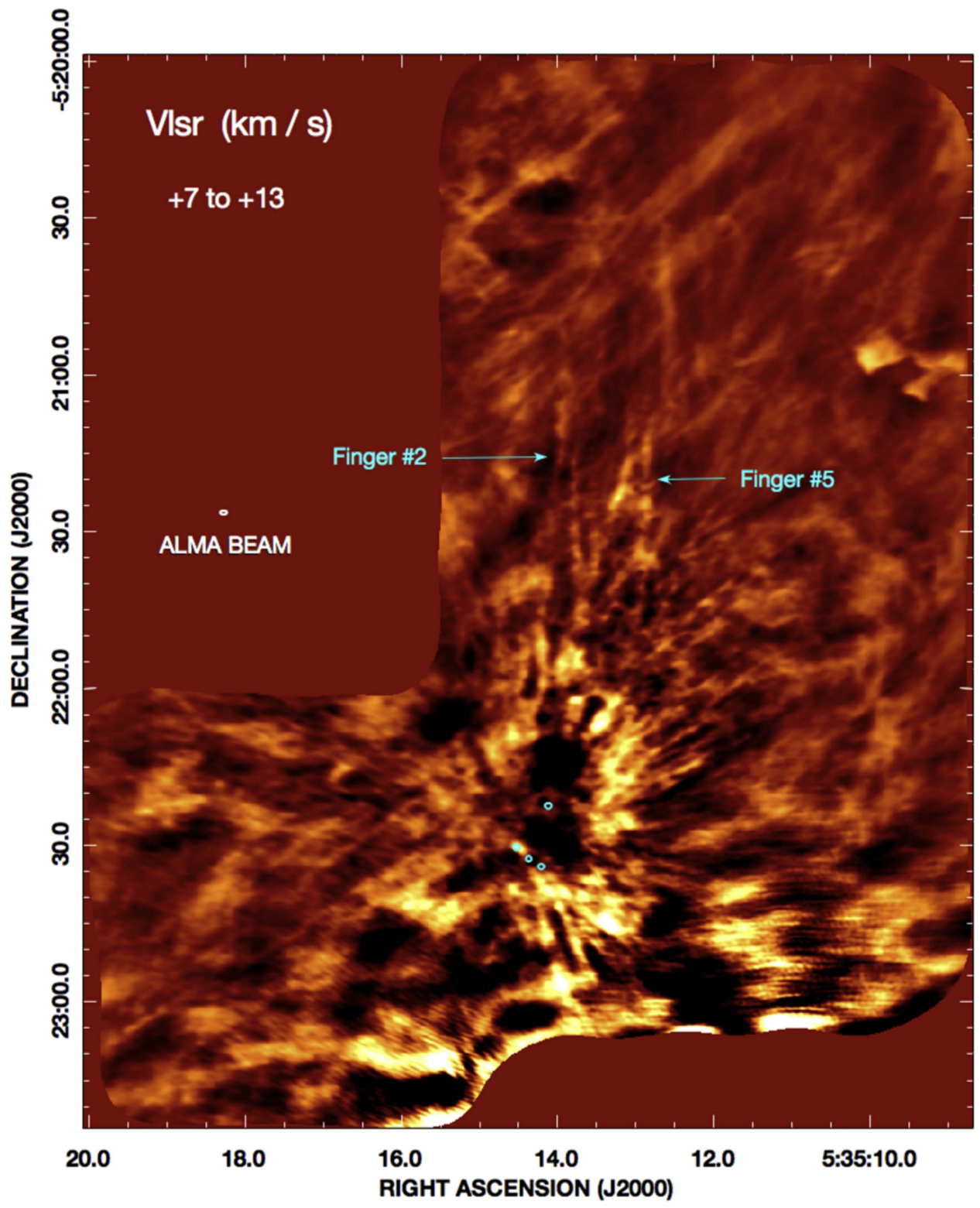

Figure 8. Integrated CO 2-1 image illustrating the small angular-scale structure from $V_{\mathrm{LSR}}=6$ to $13 \mathrm{~km} \mathrm{~s}^{-1}$. Comparison with Figures 4 and 5 shows that at these velocities, cones of $\mathrm{CO}$ emission originate from outside the prominent $\mathrm{H}_{2}$ streamers 3 and 5 . The patchy dark bays surrounding the ejection center (plus-symbol) and other parts of the image indicate missing flux due to spatial filtering of the ALMA data. Blue circles (from north to south) mark the locations of BN, Source I, Source $\mathrm{n}$, and IRc4.

most CO streamers confined to the inner $\sim 1^{\prime}$ of the ejection center, are less than $100 \mathrm{~km} \mathrm{~s}^{-1}$ similar to the $\mathrm{H}_{2}$ knot proper motions (Bally et al. 2011) and to the range of radial velocities where the brightest near-IR emission is located $(\mathrm{Oh}$ et al. 2016). The factor of two larger motions in the north and northwest $\mathrm{H}_{2}$ and [Fe II] fingers and $\mathrm{HH}$ objects suggests that a fan of unusually fast ejecta was launched along the ejection direction of $\mathrm{BN}$.

Figures 11 through 17 show that the northern $\mathrm{H}_{2}$ fingers lack $\mathrm{CO}$ emission. It is possible that some $\mathrm{CO}$ close to the radial velocity of Orion A is obscured by the foreground cloud. Furthermore, in the presence of bright $\mathrm{CO}$ emission near the rest velocity of Orion A and within tens of arcseconds of OMC1, where emission extends over a wide range of velocities, residual beam artifacts from incomplete cleaning of the data and missing flux resolved out by the interferometer may hide emission below $10 \mathrm{~K}$. However, toward the northern
$\mathrm{H}_{2}$ fingers, the noise in the spectral-line maps is less than 5 $\mathrm{mJy} /$ beam (away from the $\sim 7$ to $12 \mathrm{~km} \mathrm{~s}^{-1}$ radial velocity range, where $\mathrm{CO}$ emission from Orion $\mathrm{A}$ is bright). Emission has to be fainter than $0.1 \mathrm{~K}$ to be below our sensitivity.

Figure 7 shows that $\mathrm{H}_{2}$ streamer \#1 is aimed directly toward HH 210, which is dominated by [Fe II] emission with very little associated $\mathrm{H}_{2}$. $\mathrm{HH} 210$ exhibits the highest proper motions, 380 to $425 \mathrm{~km} \mathrm{~s}^{-1}$ (Doi et al. 2002), and contains $\sim 10$ megaKelvin plasma observed by the Chandra X-ray telescope (Grosso et al. 2006). HH 210 has a radial velocity of $-64 \mathrm{~km} \mathrm{~s}^{-1}$ (Doi et al. 2004) implying that it moves close to the place of the sky. There are no CO features at any radial velocity that convincingly point toward this high-velocity shock from OMC1.

HH 201, one of the brightest shocks associated with the OMC1 explosion at visual wavelengths, and along with HH 210, among the two brightest [Fe II] dominated shocks in 


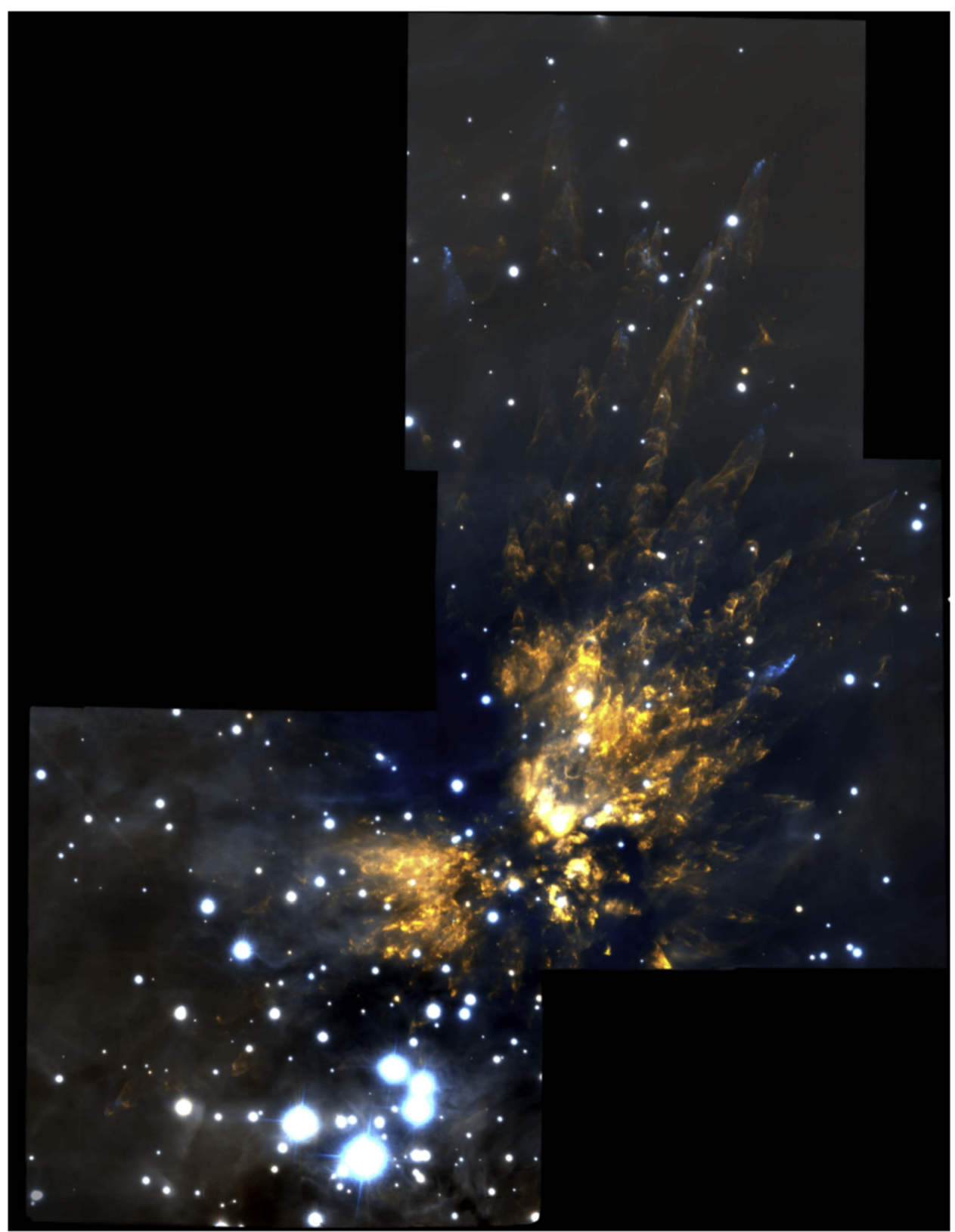

Figure 9. OMC1 outflow in the $2.12 \mu \mathrm{m} \mathrm{H}_{2}$ (orange) and $1.64 \mu \mathrm{m}$ [Fe II] (blue) lines in the near-infrared. This image was obtained with the Gemini South Adaptive Optics Imager (GSAOI) and the Gemini Multi-conjugate adaptive optics System (GeMS) on the Gemini South $8 \mathrm{~m}$ telescope that uses five Sodium lasers to generate artificial guide stars. The angular resolution of these observations is about 0!.06. Taken from Bally et al. (2015).

the Orion Nebula, also has no clear CO counterpart. Doi et al. (2004) found a radial velocity of -260 to $-284 \mathrm{~km} \mathrm{~s}^{-1}$ in $\mathrm{H} \alpha$ for this object, implying that it is moving out of the cloud toward us and into the Orion Nebula. HH 201 lies in the region of the northwest-facing bubble seen at both red- and blueshifted $\mathrm{CO}$ radial velocities. There is some low velocity $\mathrm{CO}$ at blueshifted radial velocities with respect to OMC1 at $V_{\mathrm{LSR}} \approx 2.4$ to about $5.3 \mathrm{~km} \mathrm{~s}^{-1}$ in the northwest bubble several arcseconds beyond the tip of HH 201.

Although no $\mathrm{CO}$ streamers reach the ends of the north and northwest fingers, the bases of several of the most prominent $\mathrm{H}_{2}$ fingers contain clumpy, low-velocity $\mathrm{CO}$ with peak brightness temperatures ranging from $10 \mathrm{~K}$ to less than 0.5 $\mathrm{K}$. Figure 11 shows the summed emission from $V_{\mathrm{LSR}}=-10$ to $0 \mathrm{~km} \mathrm{~s}^{-1}$ at blushifted radial velocities (in cyan) superimposed on the redshifted emission at $V_{\mathrm{LSR}}=+21$ to $34 \mathrm{~km} \mathrm{~s}^{-1}$ (in red). Clumps of $\mathrm{CO}$ at both red and blueshifted radial velocities are associated with fingers \#2, \#3, \#4, and \#5 (Figure 7). Figure 12 shows the summed emission from $V_{\mathrm{LSR}}=+1$ to $+7 \mathrm{~km} \mathrm{~s}^{-1}$ at blueshifted radial velocities (in cyan) with respect to the OMC1 rest frame, superimposed on the redshifted emission at $V_{\mathrm{LSR}}=+13$ to $+20 \mathrm{~km} \mathrm{~s}^{-1}$ (in red). In this figure, only streamers \#3 and \#5 are traced by CO. The widths of the $\mathrm{CO}$ emission orthogonal to the $\mathrm{H}_{2}$ features are larger than in the previous figure. Figures 13-17 show progressively higher velocity $\mathrm{CO}$ emission integrated over the radial velocities indicated in the figures from $V_{\mathrm{LSR}}=-10$ to $+34 \mathrm{~km} \mathrm{~s}^{-1}$. These figures suggest that the longest north and northwest $\mathrm{H}_{2}$ fingers most likely lie in the plane of the sky.

Figures 11 and 12 show CO clumps associated with a CO streamer aimed east of streamer \#1. This feature is only associated with very faint $\mathrm{H}_{2}$ and [Fe II] emission with the 


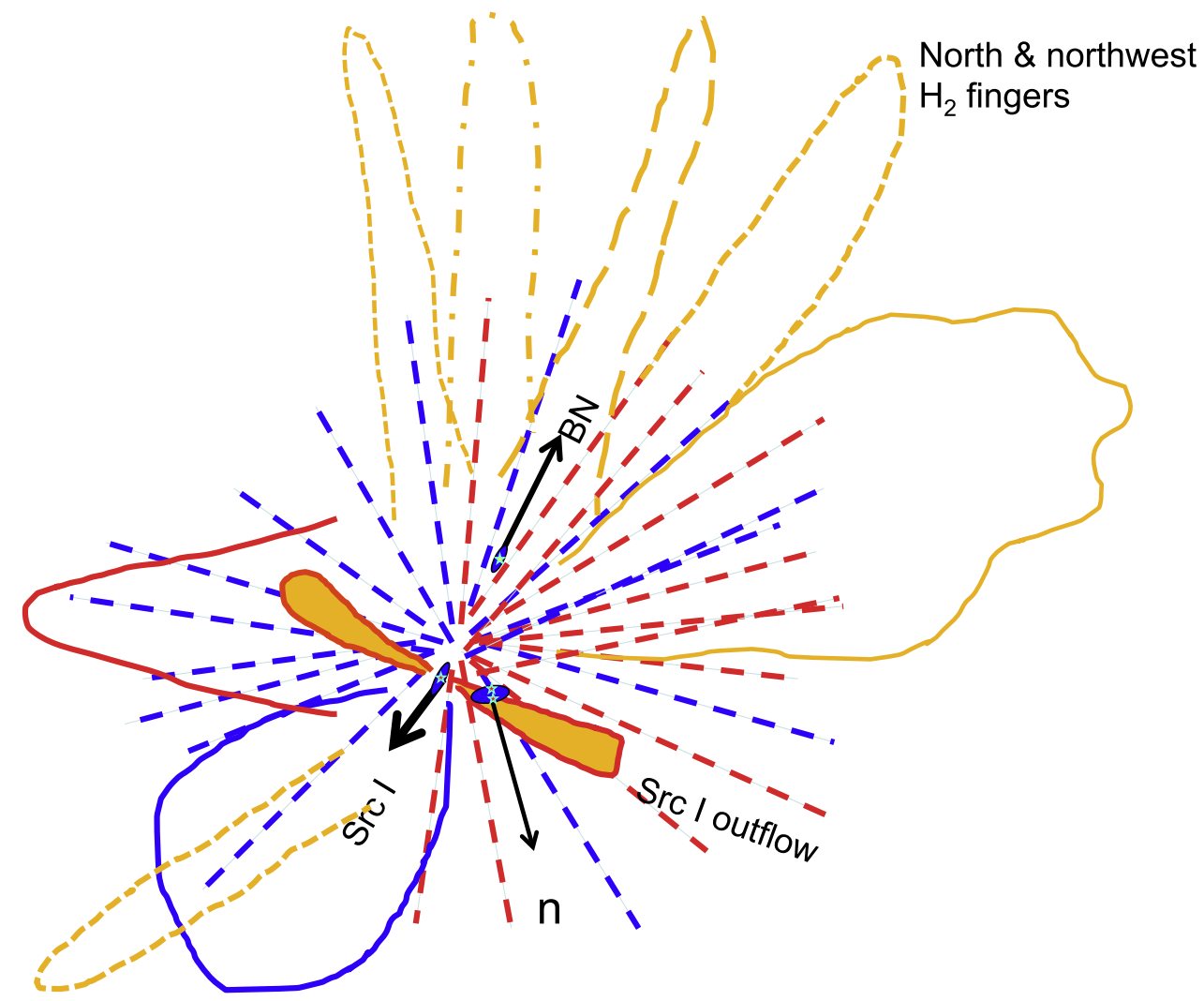

Figure 10. Cartoon showing the major elements of the OMC1 outflow. Dashed red and blue lines illustrate the high-velocity streamers. Dashed yellow-orange lines mark the locations of the most prominent $\mathrm{H}_{2}$ fingers; the solid orange line shows the bubble of low-velocity red- and blueshifted ejecta fan toward the northwest. Solid red and blue lines show the red- and blueshifted bubbles of ejecta extending to the east and southeast. The solid orange region bounded by a solid red line shows the young Source I flow. The relative locations of Source I, n, and BN, their proper motions, and suspected orientations of their disks are also illustrated, but not to scale.

brightest feature located at the east edge of the mapped field at 05:35:15.73, $-5: 21: 12.6$ at the left edge of Figures 13-16 about one-third of the way from the bottom. This streamer and its associated shocked emission may be partially hidden by dust ridge extending north-northeast from OMC1 toward OMC2.

Several models might explain the gaps between the ends of the $\mathrm{CO}$ streamers and the near-IR shocks in the fingertips. First, above the velocity of the order of $\sim 100 \mathrm{~km} \mathrm{~s}^{-1}$ observed at the leading edges of many streamers, shocks may completely destroy CO. Second, the fastest ejecta may have been launched in mostly atomic or ionized form and may not have had sufficient time, or the conditions, to form molecules. If either model is correct, the gap between the fingertips and the $\mathrm{CO}$ streamer ends is expected to be filled with dense atomic gas or plasma; tracers such as sub-millimeter $\mathrm{CI}$, far-IR $\mathrm{C}^{+}$, or radio continuum should detect it. Third, the gap may be empty, with dense clumps located close to the fingertips that are required to drive the observed high-proper motion shocks. Future observations will distinguish between these scenarios. Figure 10 shows a cartoon illustrating the major observed features of the OMC1 outflow, the relative locations and motions of the ejected stars, and the suspected orientations of their disks.

\subsection{Other Outflows in the Field}

Several protostellar outflows unrelated to OMC1 are also seen in the CO data. Teixeira et al. (2016) surveyed the field northeast of the region covered by our observations in the $1.3 \mathrm{~mm}$ bands. They found a number of compact millimeter continuum sources and molecular outflows. SMA-15 appears at the left edge in our maps and drives a prominent bubble and bipolar high-velocity outflow launched in an east-west direction. The western, redshifted lobe of this flow overlaps with $\mathrm{HH} 210$. A small west-facing $\mathrm{H}_{2}$ bow shock is located along the axis of this flow about $10^{\prime \prime}$ west of SMA-15. A complex, redshifted outflow lobe can be traced for nearly $90^{\prime \prime}$ to the west and northwest and appears to be deflected in this direction.

Teixeira et al. (2016) SMA-14, located just beyond our mapped field about $25^{\prime \prime}$ north of SMA-15, drives a highly collimated, blueshifted $\mathrm{CO}$ jet at $\mathrm{PA} \approx 225^{\circ}$. In Figure 11 , this flow can be seen as a $10^{\prime \prime}$-long cavity with a width of about $5^{\prime \prime}$. This flow is pointed slightly south of a collection of CO knots and an $\mathrm{H}_{2}$ bow facing toward the southwest. If the collection of $\mathrm{CO}$ knots and the $\mathrm{H}_{2}$ bow are powered by the SMA-14 outflow, then this flow must also experience a small northward deflection.

A blueshifted, southeast-northwest oriented filament in the upper left corner of Figure 11 traces back to Teixeira et al. (2016) SMA-18 or possibly SMA-17 beyond the east edge of our data. This feature may trace a highly collimated jet.

Finally, in a small blushifted outflow emerging from the OMC1-S region ( $\sim 90^{\prime \prime}$ south of OMC1) is seen in the lower right corner of Figure 2. This flow appears to be a collimated, knotty jet between $V_{\mathrm{LSR}} \approx-8$ to $+4 \mathrm{~km} \mathrm{~s}^{-1}$. This flow may be related to HH 625 (Kaifu et al. 2000; O’Dell et al. 2015).

\section{Discussion}

Figures 1 through 4 show that the high-velocity CO streamers point pack to a location between the three ejected 


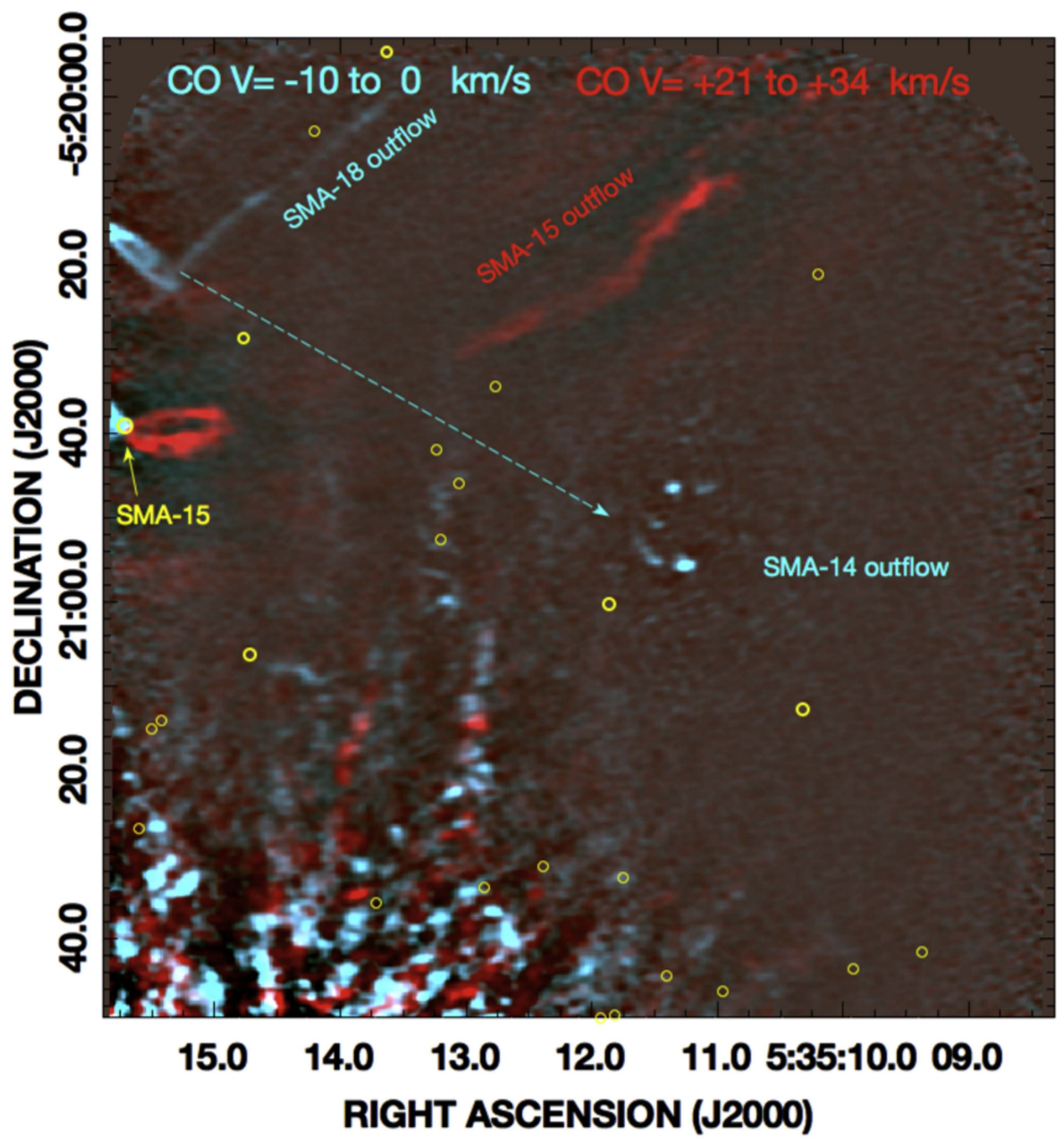

Figure 11. Image showing the $\mathrm{CO}$ emission at $V_{\mathrm{LSR}}=-10$ to $0 \mathrm{~km} \mathrm{~s}^{-1}$ in cyan and +21 to $+34 \mathrm{~km} \mathrm{~s}^{-1}$ in red in the region containing the north and northwest fingers. The yellow circles mark compact (unresolved) $1.3 \mathrm{~mm}$ continuum sources. Some can be identified with stars in visual or near-IR images; others, such as SMA15 (Teixeira et al. 2016) have no visual or near-IR counterparts. The outflows unrelated to OMC1 are indicated. SMA-15 drives a bipolar flow with a redshfted lobe deflected toward the north. The blueshifted limb-brightened cavity marks an outflow, possibly originating form SMA-14 in Teixeira et al. (2016). This flow appears to terminate in the compact knots just east of the label "SMA-14 outflow." These knots are associated with a southwest-facing bow shock in $\mathrm{H}_{2}$ that is also an $\mathrm{HH}$ object. Finally, the highly collimated blueshifted filament labeled "SMA-18 outflow" traces a jet, possibly from SMA 18 in Teixeira et al. (2016). These flows are described in the text.

stars. Over $80 \%$ of the streamer orientations trace back to within an elliptical region with major and minor axis diameters of $\sim 3 \times 5^{\prime \prime}$ oriented at $\mathrm{PA} \sim 330^{\circ}$ and centered at $05: 35: 14.33,-5: 22: 27.8$, shown as a cross in the figures and close to the suspected ejection site of the OMC1 runaway stars when the proper motions are corrected to the reference frame of OMC1 as discussed below. The coincidence between the streamer orientations and the site of stellar ejection indicates that the explosion in the gas was likely powered by the stellar ejection. As discussed below, the energetics requires an auscale encounter between a pair of $10 M_{\odot}$ stars, or a sub-au-scale encounter between less massive stars. The fastest ejecta can be created by the gravitational slingshot of circumstellar or stellar photospheric material as three or more stars approach each other at speeds of the order of the Kepler speed given by their masses and separations. Dense gas in OMC1 may have reshaped the fast ejecta into the observed Hubble flow streams as discussed in the next section. Following the ejection of the stars, orbital motion of material bound to the cluster prior to ejection would be converted into linear expansion to produce low velocity ejecta. Numerical simulations of collisions or near misses show that debris is likely to be preferentially ejected along the orbital planes of the interacting stars during the close encounter (Portegies Zwart \& van den Heuvel 2016). The bubbles and fans of low-velocity ejecta noted above may have their origins in such streams. The masses and momenta of the ejected stars are critical parameters that must be considered by any model of the OMC1 explosion.

\subsection{The Streamers}

The high-velocity $\mathrm{CO}$ in $\mathrm{OMC} 1$ consists of a nearly isotropic distribution of over 100 linear streamers whose radial velocities increase with projected distance from the source. Toward the northeast and southwest, the CO streamers and the distance of the $\mathrm{H}_{2}$ and [Fe II] shocks in the Orion fingers have comparable spatial extent. However, toward, the north and northwest, the distance of the $\mathrm{CO}$ streamers from the ejection region is about one-half of the distance of the most distant $\mathrm{H}_{2}$ fingers and [Fe II] fingertips. The largest $\mathrm{CO}$ radial velocities are also about 




Figure 12. Image showing the $\mathrm{CO}$ emission in the region containing the north and northwest fingers showing emission at $V_{\mathrm{LSR}}=+1$ to $+7 \mathrm{~km} \mathrm{~s}{ }^{-1}$ in cyan and +13 to $+20 \mathrm{~km} \mathrm{~s}^{-1}$ in red. Yellow circles are as in Figure 11.

two times smaller than the fastest measured proper motions of the $\mathrm{H}_{2}$ and [Fe II] shocks. The $\mathrm{CO}$ morphology resembles the jet-like features seen in high-speed videos of powerful terrestrial explosions occurring inside a damping medium. Experiments with explosives embedded in the center of a sphere of solid beads, wet-beads, or liquids produce hundreds of jet-like streamers with ejection speeds increasing with distance and a morphology similar to those seen in Orion (Frost et al. 2012; Milne et al. 2016; for videos, see https://www. youtube.com/watch? $v=7814 \mathrm{cU} 5 \mathrm{~F} 2$ YE). Particle jets displaying a Hubble-law velocity-distance relation are a general characteristic of explosions occurring in an inert damping medium.

Stone et al. (1995) proposed that bullets responsible for driving the multiple $\mathrm{H}_{2}$ fingers and [Fe II] fingertips in the OMC1 outflow were produced by Rayleigh-Taylor (RT) hydrodynamical instabilities resulting from the interaction of a wind with the surrounding medium. A swept-up shell is subject to RT instabilities if it accelerates; it can be triggered by a wind with increasing mechanical luminosity, a steady wind running into a medium with a steeper than inverse square-law density profile, or a combination of increasing wind power and a shallower density profile. RT instabilities can form dense clumps whose wakes create low-excitation $\mathrm{H}_{2}$ fingers and high-excitation $[\mathrm{Fe}$ II] fingertips. McCaughrean \& Mac Low (1997) proposed a variant of this model in which multiple sources in a dense cluster lead to the formation of a fragmented shell.

Ejecta from an explosion occurring inside a cocoon of relatively stationary material will be decelerated by interactions with the medium. While the cores of sufficiently dense or massive clumps may retain much of their initial velocities as they move through the medium and exit its outer boundary, the motions of less massive or lower density clumps will be damped. Furthermore, the clump surface layers will be shorn-off, mixed with the medium, decelerated, and subjected to KelvinHelmholtz (KH) shear instabilities. Assuming that the cocoon has an outer boundary, the passage of sufficiently massive ejecta through the medium will result in the emergence of a stream of debris having a distribution of masses and a wide range of velocities. In the absence of further deceleration, the ejecta will be sorted by velocity upon exiting the damping medium of the cocoon. A finite time later, the distance travelled will be proportional to the speed of ejecta as it leaves the cocoon, forming a Hubble flow as observed in the OMC1 explosion. 


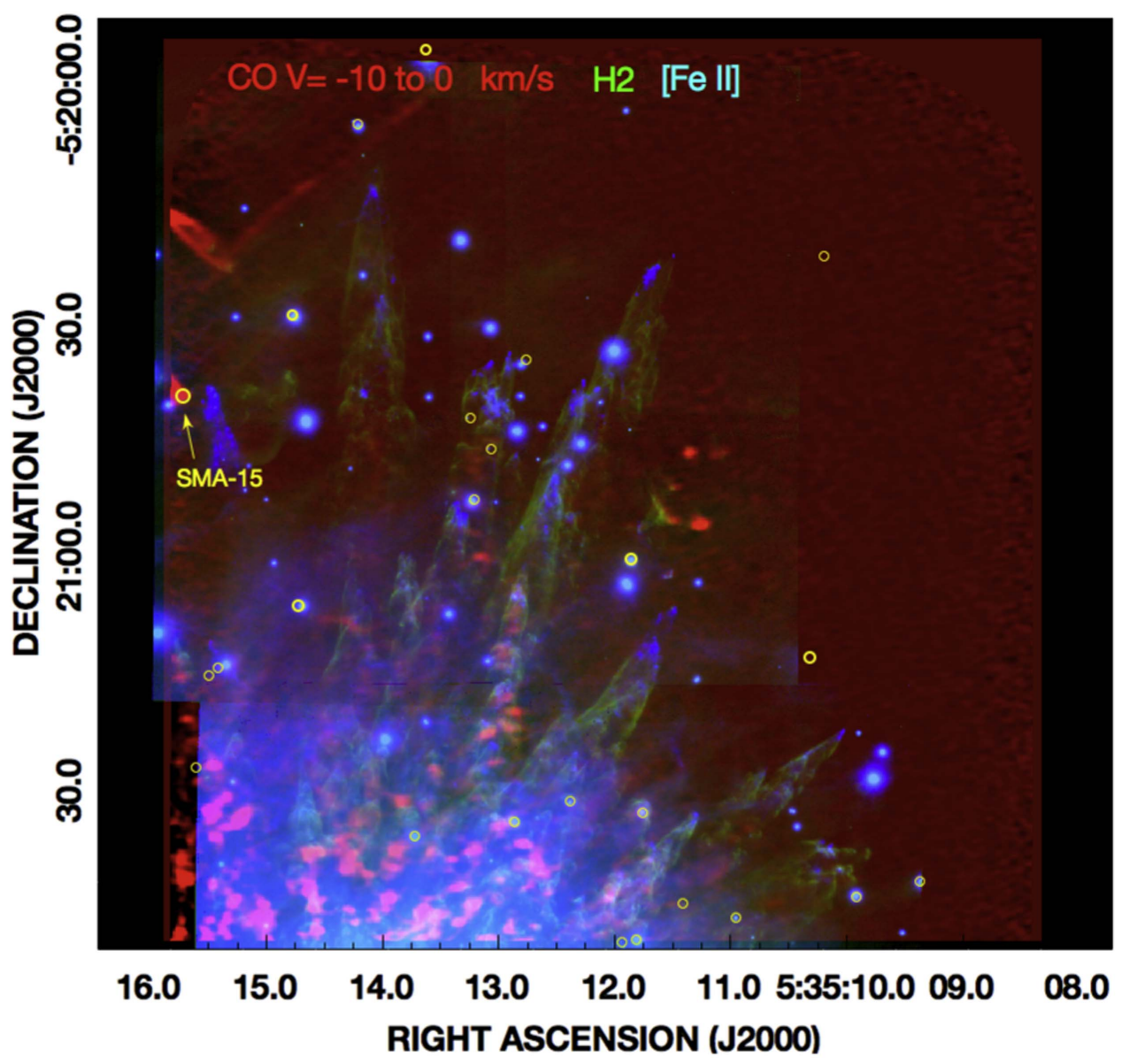

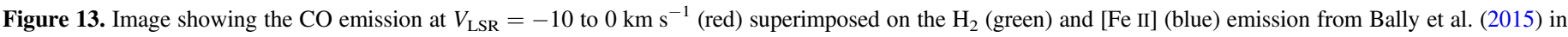
the region containing the north and northwest fingers.

\subsection{The Masses of the OMC1 Runaway Stars}

Linking the OMC1 explosion to the dynamic ejection of its runaway stars requires knowledge of the stellar masses. The mass of the fastest ejected star, BN, has been estimated to be between 12 and $15 M_{\odot}$ (Scoville et al. 1983) or 8 to $10 M_{\odot}$ (Tan 2004; Chatterjee \& Tan 2012). Assuming that Source I has an oppositely directed momentum, it must have a mass of 16-25 $M_{\odot}$ (Goddi et al. 2011b). Source n, also involved in the dynamic interaction, has a mass of $\sim 3$ to $6 M_{\odot}$. Thus, to conserve momentum in a three-way interaction involving Source $n$, Source I should have to have a mass of $\sim 10-22 M_{\odot}$ to balance the momentum of $\mathrm{BN}$.

Millimeter and sub-millimeter interferometric measurements of the rotation curve of the $50 \mathrm{au}$ radius disk surrounding source I indicate a mass between only 5 and $7 M_{\odot}$ (Matthews et al. 2010; Plambeck \& Wright 2016). If this estimate is correct, then Source I plus n by themselves could not have been responsible for launching the $\mathrm{BN}$ object with a velocity of $\sim 26 \mathrm{~km} \mathrm{~s}^{-1}$ and the model proposed by Tan (2004) in which $\mathrm{BN}$ was ejected from the Trapezium $\sim 4000$ years ago may be more viable. However, the ejection of $\mathrm{BN}$ by the Trapezium leaves unexplained the $10-14 \mathrm{~km} \mathrm{~s}^{-1}$ motions of Source I toward the southeast and motion of Source $n$ from the ejection center of the Orion fingers and $\mathrm{CO}$ streamers in a direction opposite to the motion of BN. Furthermore, a passage of BN to OMC1 sufficiently close to trigger an explosion is unlikely. Using the projected separation of $\theta^{1}$ Ori $\mathrm{C}$, the most massive member of the Trapezium group of massive stars in the core of the Orion Nebula, and BN of $60^{\prime \prime}(\sim 25,000 \mathrm{au})$, an encounter within a radius of 50 au of Source I has a probability of $\sim 10^{-6}$, assuming an isotropic probability distribution of the ejection direction of BN. However, Chatterjee \& Tan (2012) argue that such an event is not impossible and that the passage of $\mathrm{BN}$ through OMC1 could have triggered the explosion observed in the gas by driving a major accretion event onto one of the OMC1 massive stars.

As discussed by Matthews et al. (2010) and Plambeck \& Wright (2016), it is possible that the Source I disk rotation curve underestimates the central star's mass because of internal pressure support. If magnetic fields or turbulence is sufficiently strong, the disk would rotate at sub-Keplerian velocities (Shu et al. 2008). Additionally, a strong outflow driven by magnetocentrifugal acceleration exerts a strong braking torque on the disk surface. Thus, the observed velocities may underestimate the midplane orbital velocities, and therefore the mass of the central star.

Plambeck \& Wright (2016) found that the Source I disk has a mass of 0.02 to $0.2 M_{\odot}$ and a projected thickness of about $20 \mathrm{au}$. Using the $50 \mathrm{au}$ radius, these masses imply that the mean density of $\mathrm{H}_{2}$ ranges from 1.7 to $17 \times 10^{10} \mathrm{~cm}^{-3}$. Girart et al. (2004) and Plambeck et al. (2003) found evidence for a magnetic field in Source I. Using the Crutcher (2012) scaling of magnetic field strength with density, $B \sim 10\left(n / 300 \mathrm{~cm}^{-3}\right)^{2 / 3}$ micro-Gauss, where $n$ is the $\mathrm{H}_{2}$ volume density, the magnetic field strength in the Source I disk could be 


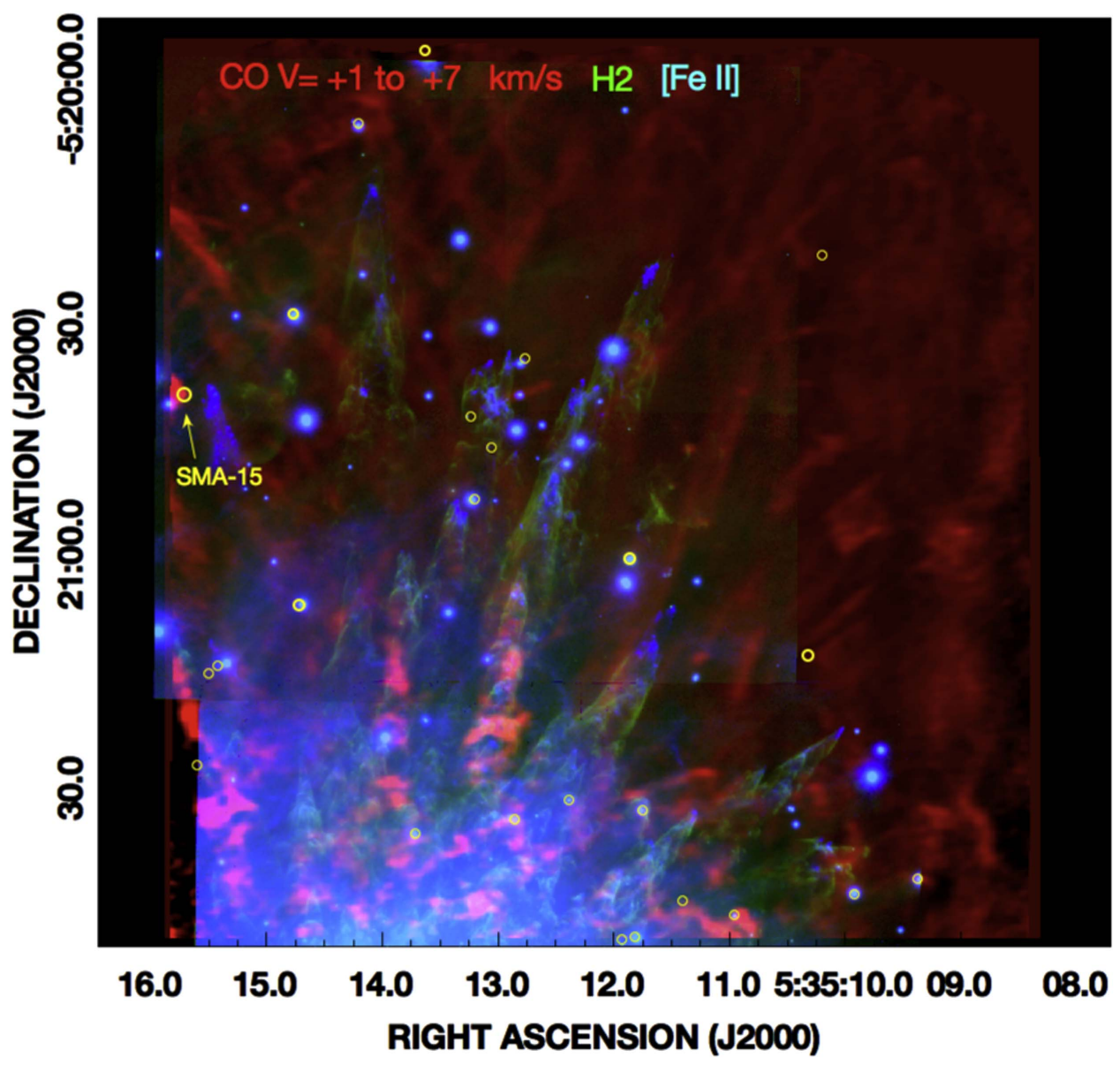

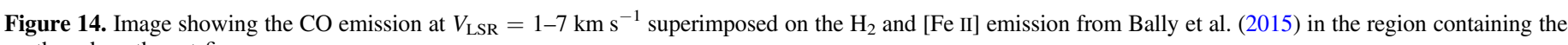
north and northwest fingers.

around 1 to 10 Gauss for the above densities. The total magnetic energy of the Source I disk would then be $E_{B} \approx\left(B^{2} / 8 \pi\right) \pi R_{\text {disk }}^{2} Z_{\text {disk }} \sim 2 \times 10^{43}$ to $2 \times 10^{45}$ erg. Here, $R_{\text {disk }}$ is the observed disk outer radius and $Z_{\text {disk }}$ is its vertical extent. The kinetic energy in orbital motion of the 0.02 to $0.2 M_{\odot}$ disk with a characteristic orbit speed of $10 \mathrm{~km} \mathrm{~s}^{-1}$ is about $10^{43}-10^{45} \mathrm{erg}$, depending on the mass distribution. Because the magnetic energy and disk orbital kinetic energy may be comparable, magnetic breaking and sub-Keplerian disk rotation is plausible. Additionally, the young, thermal $\mathrm{SiO}$ and $\mathrm{H}_{2} \mathrm{O}$ maser flow emerging from Source I contains the brightest and highest density portion of the OMC1 outflow. Thus it is likely to exert a substantial torque on the Source I disk: the rotation-curve-based mass estimates for Source I may be lower bounds.

A recent dynamical interaction and abrupt acceleration of Source I to a velocity of $V_{*} \sim 13 \mathrm{~km} \mathrm{~s}^{-1}$ would have disrupted any pre-existing outer disk beyond the gravitational radius, $R_{G} \approx G M / V_{*}^{2}=148$ au for $M=22 M_{\odot}$, or $34 \mathrm{au}$ for $M=5 M_{\odot}$. Disk material closer to the star than $R_{G}$ could remain gravitationally bound following acceleration by the dynamical interaction. However, as discussed below, acceleration to these velocities requires an au-scale encounter with the other stars. Such a penetrating encounter would eject most of the disk beyond $R_{G}$ and would severely perturb the remainder inside this radius. A deep, penetrating encounter leading to the acceleration of the interaction-formed binary or merger remnant would likely lead to a re-orientation of the angular momentum vector of the surviving disk (Moeckel \& Bally 2007a; Moeckel \& Goddi 2012). The damping-time would be a few times the orbit time at the $\sim 50$ au outer radius of retained disk material, or longer than 75-160 years for $M=22-5 M_{\odot}$ stars, respectively. Material ejected by such close encounters that did not reach escape speed could be falling-back onto the remnant disk for many times the orbit speed at the disk outer radius, producing longer-lasting disk perturbations. Additionally, if the interaction resulted in the formation of a compact binary, the orbits are likely to be highly eccentric, and non-coplanar with the surviving disk. Gravitational forces from inclined, eccentric orbital motion would stir the disk and drive turbulence.

Thus, it is possible that torques exerted by a powerful outflow, magnetic fields, and internal turbulence, contribute to sub-Keplerian rotation of the Source I disk, leading to an underestimate of the mass of Source I.

\subsection{The Potential Role of Source $n$}

The fast proper motion of $\mathrm{BN}$ and the slow-motion of Source I could be partially reconciled with the recent measurement of the motion of Source $n$ by Dzib et al. (2016). The momentum and kinetic energy in the motion of BN could be balanced by slower and lower-mass Source I plus the momentum and energy of Source $\mathrm{n}$. Source $\mathrm{n}$ has a luminosity of $\sim 2000 L_{\odot}$, an estimated mass of $3-6 M_{\odot}$, and is surrounded by a 340 by 230 au diameter disk detected at 8 and $11.7 \mu \mathrm{m}$ 


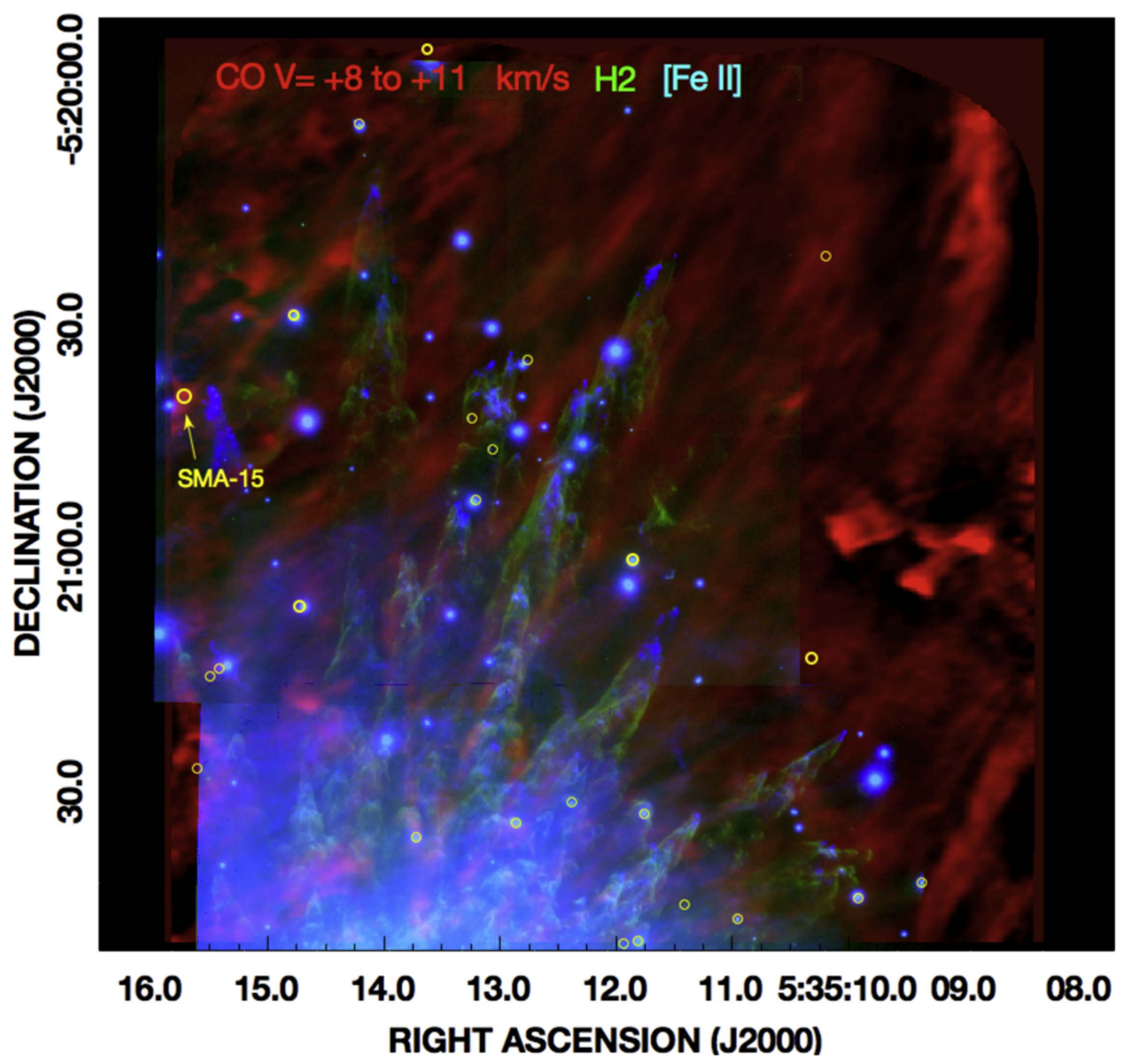

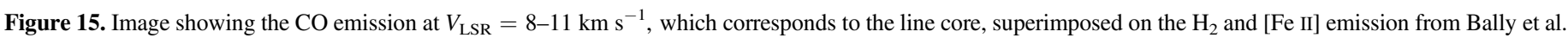
(2015) in the region containing the north and northwest fingers.

(Greenhill et al. 2004). The Source $\mathrm{n}$ disk emits in the $2.3 \mu \mathrm{m}$ overtone bands of CO (Luhman et al. 2000). A pair of radio sources and a jet-like feature extending to the southwest at PA $\sim 200^{\circ}$ indicate that it may be a binary YSO that powers a one-sided jet roughly orthogonal to the disk (Dzib et al. 2016). Recently Dzib et al. (2016) detected a $15.4 \mathrm{~km} \mathrm{~s}^{-1}$ proper motion of the compact binary core of the radio image toward the south. All three of the OMC1 radio sources, Source n, BN, and Source I were within 110 au of each other in the year AD $1475 \pm 6$ (Dzib et al. 2016). Source n, along with BN and Source I may have played an important role in the dynamics of the OMC1 about 540 years ago.

A proper motion of $\sim 15 \mathrm{~km} \mathrm{~s}^{-1}$ implies that the trajectory of Source $\mathrm{n}$ would have intersected the trajectory of Source I between 370 and 540 years ago; if the proper motion were $26 \mathrm{~km} \mathrm{~s}^{-1}$ as suggested by Gómez et al. (2008), the intersection would have occurred 270 and 345 years ago. In the model in which a dynamical decay launched Source I and BN $\sim 540$ years ago, Source $\mathrm{n}$ was either ejected during this interaction, several hundred years after the main event, or it is yet another moving star whose origin is unrelated to the event that launched the massive stars. It is possible that the event 500 years ago involved four or more stars in which the interaction produced an unstable triple or quadruple moving in the opposite direction to BN. This system could have decayed up to a few hundred years later and launched Source I and Source $\mathrm{n}$ on their current trajectories. Such a staged disintegration could explain the several arcsecond dispersion in the streamer orientations. In this model, either Source I, Source n, or both would have to be a compact binary or merger remnant (Reipurth \& Mikkola 2012, 2015).

The sum of the 5-7 $M_{\odot}$ mass of Source I from (Plambeck \& Wright 2016) and the 3-6 $M_{\odot}$ mass of Source $\mathrm{n}$ is still at most $13 M_{\odot}$. Assuming these masses, and using the stellar speeds in our assumed OMC1 reference frame, and adding the momenta of these two ejected stars give a total momentum of 98-164 $M_{\odot} \mathrm{km} \mathrm{s}^{-1}$, a factor of nearly two to three less than the $230-430 M_{\odot} \mathrm{km} \mathrm{s}^{-1}$ momentum of BN. No reasonable shift of the $\mathrm{OMC1}$ rest frame can bring these momenta into agreement. Thus, either the mass of Source I or $\mathrm{n}$ has been severely underestimated, or there is another ejected massive member that has not yet been identified. Other massive objects that might have participated in the interaction include other stars, or high density and massive compact cores such as IRc4.

\subsection{The Energetics of the OMC1 Explosion}

It is possible that the OMC1 core has a net motion of a few $\mathrm{km} \mathrm{s}^{-1}$ with respect to the stars in the Trapezium region. Within $1^{\prime}$ of $\mathrm{OMC} 1$, the radial velocity of molecular gas increases from about 8 to $11 \mathrm{~km} \mathrm{~s}^{-1}$ from south to north. The gravitational potentials of the Trapezium cluster and OMC1, each of which contains a mass of around $100 M_{\odot}$ and are separated by about $0.1 \mathrm{pc}$, could produce a relative motion of the order of 3-4 $\mathrm{km} \mathrm{s}^{-1}$, comparable to the observed change in radial velocity of the molecular tracers in the vicinity of OMC1. 


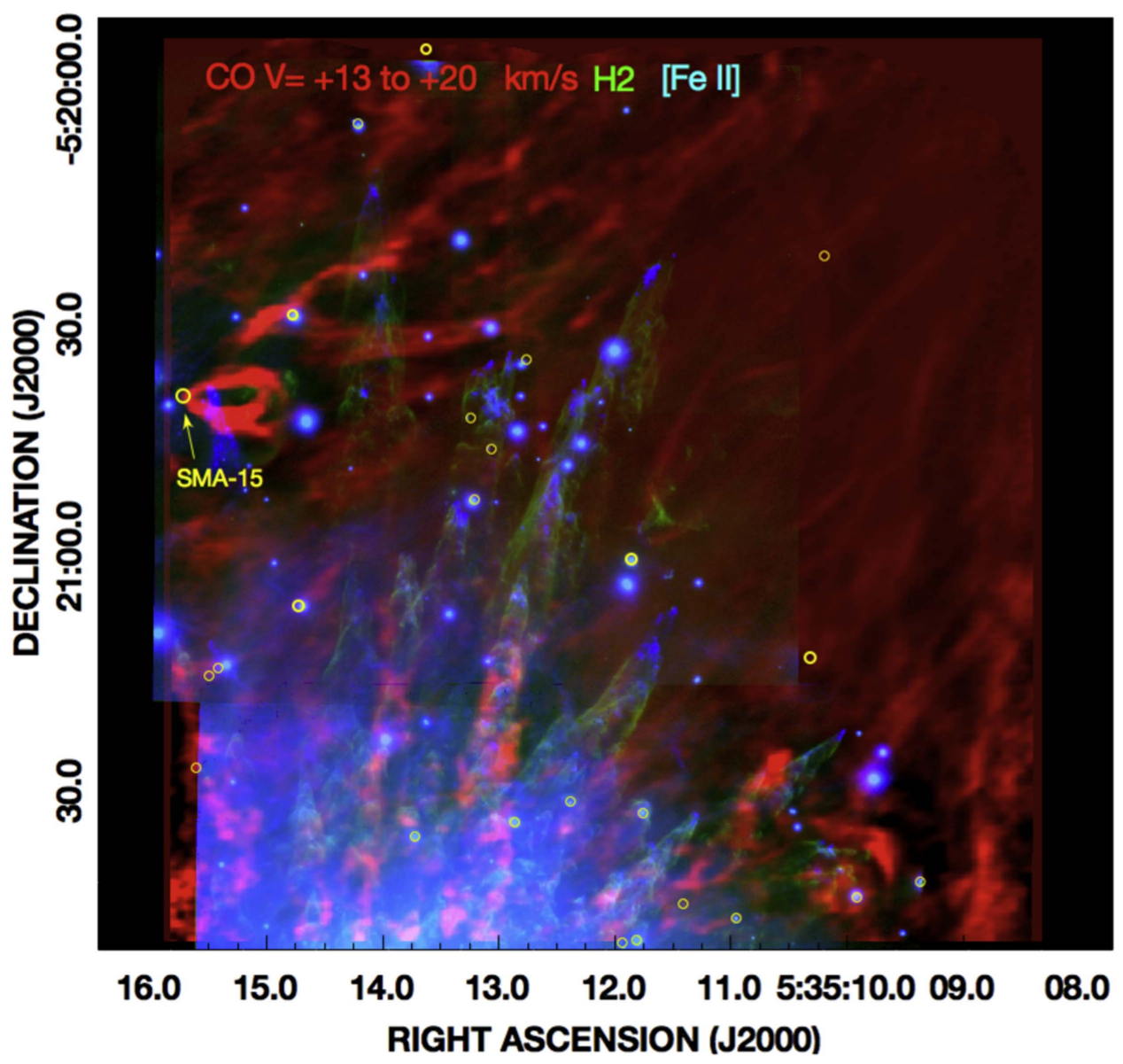

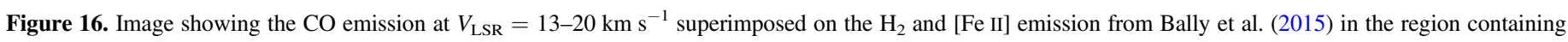
the north and northwest fingers.

The Dzib et al. (2016) proper motions, referenced to a frame in which 79 Orion radio sources are at rest, are shown as solid vectors in Figures 1, 2, and 4. These vectors intersect several arcseconds east of the ejection center indicated by the earlier measurements and significantly displaced from the center of mass of the three stars assuming mass ratios of 4:2:1 for Source $\mathrm{I}, \mathrm{BN}$, and Source $\mathrm{n}$, respectively. This location is also several arcseconds east of the point where a traceback of the $\mathrm{CO}$ streamers intersects. The dashed proper motion vectors in Figures 1, 2, and 4 were derived by assuming that OMC1 is moving west with respect to the Trapezium stars with a velocity of $4 \mathrm{~km} \mathrm{~s}^{-1}$ toward $\mathrm{PA}=273^{\circ}$. Such a motion brings the ejection center to coincide with the center of mass of the stars assuming the above mass ratios.

The proper motions of BN, Source I, and Source $n$ were discussed in the Introduction. The radial velocity of Source $n$ is unknown. However, given that it is visible in the near-IR, it must suffer less extinction than the nearby Source I, so it may be on the nearside of OMC1 and likely blueshifted. The lower speed of Source I, combined with the $5^{\circ}-10^{\circ}$ estimated error in the orientation of its proper motion may account for the differences in its ejection direction measured by the various groups. The proper motions of BN, Source I, and $\mathrm{n}$ in the OMC1 reference frame discussed above are 27, 12, and $17 \mathrm{~km} \mathrm{~s}^{-1}$, respectively. Combining the radial velocities with the proper motions, and assuming that the line-of-sight velocity of Source $\mathrm{n}$ is $-5 \mathrm{~km} \mathrm{~s}^{-1}$, the total speed of Source BN, Source $\mathrm{I}$, and $\mathrm{n}$ are about 27,13 , and $17 \mathrm{~km} \mathrm{~s}^{-1}$.
If the masses of these stars are 8-16, 10-25, and 3-6 $M_{\odot}$, respectively, the sum of the kinetic energies in the stellar motions is $E_{\text {star }} \approx(1-2) \times 10^{47} \mathrm{erg}$. The kinetic energy in the outflow today has been estimated to be $E_{\text {outflow }} \sim 4 \times 10^{46}$ (Snell et al. 1984) to $4 \times 10^{47} \mathrm{erg}$ (Kwan \& Scoville 1976). The energy source responsible for the ejection must supply at least $E_{\mathrm{tot}} \approx 10^{47} \mathrm{erg}$ during the ejection event. However, much of the initial kinetic energy of the outflow would have been converted into radiation by shock waves that render the outflow visible in $\mathrm{CO}, \mathrm{H}_{2}$, and other species. Accounting for radiative losses by shocks over the last 500 years, and the work required to climb out of an $\sim 400$ au region in the gravitational potential well of the $\sim 100 M_{\odot}$ OMC1 cloud core, the energy source responsible for the ejection must supply at least $E_{\text {tot }}=10^{48}$ erg.

The kinetic energy of the runaway stars ejected by $N$-body interactions in compact groups is thought to come from the formation or hardening of compact binary systems or stellar mergers. The formation of a binary by a three- or four-body interaction, or the interaction of a pre-existing binary with other stars that leads to the hardening of the binary (shrinking of the semimajor axis) releases gravitational potential energy, $E_{G} \approx G M_{1} M_{2} / 2 R$, where $\mathrm{R}$ is the final semimajor axis of the binary and $M_{1}$ and $M_{2}$ are the stellar masses. Assuming that Source I is such a binary (Goddi et al. 2011b), and that $M_{1}=M_{2}=10 M_{\odot}$, implies that the final binary semimajor axis must be $R \sim G M_{1} M_{2} / E_{\text {tot }}<0.9$ au to release $E_{\mathrm{tot}} \approx 10^{48} \mathrm{erg}$. 


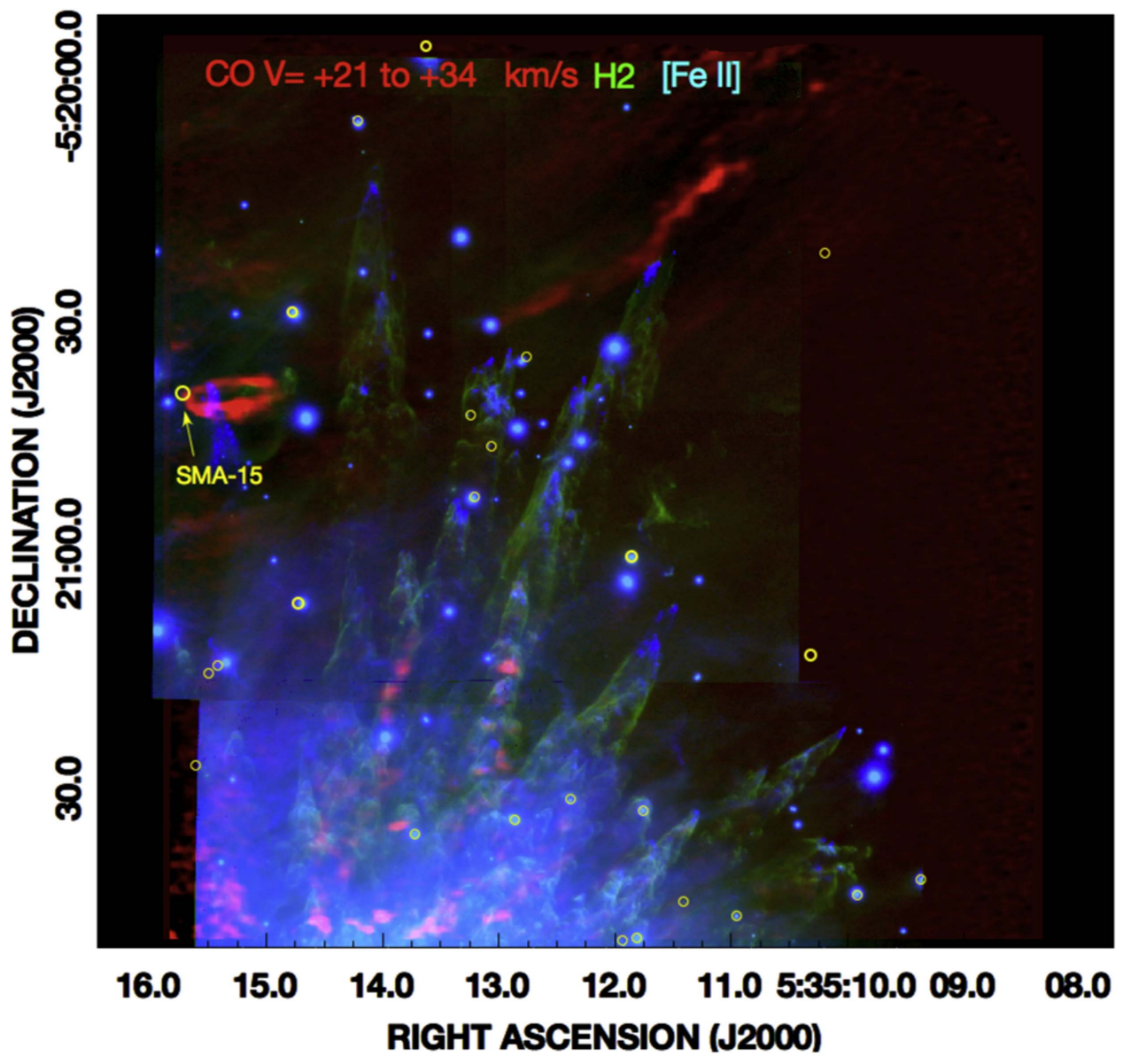

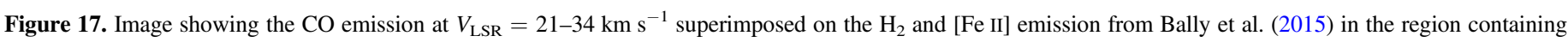
the north and northwest fingers.

The fastest ejecta in the $\mathrm{OMC1}$ outflow have speeds in excess of $400 \mathrm{~km} \mathrm{~s}^{-1}$. Although only a tiny fraction $\left(\ll 1 M_{\odot}\right)$ of the total mass has such high speeds, these speeds constrain the distance of the launch point from the source star under the assumption that the ejection velocity is comparable to the escape speed from the ejection region. Assuming a $20 M_{\odot}$ star (e.g., Source I), and no boosting of the velocities by forces other than gravity, the fastest ejecta must originate from a distance of the order of $R<3 \times 10^{12} \mathrm{~cm}(\sim 0.2 \mathrm{au})$ from the star's center of mass.

\subsection{Radio Source I: a Bloated Protostar}

Massive stars are thought to accrete at high rates from their parent cloud cores. To form a star with a mass of $10 M_{\odot}$ in $10^{5}$ years requires an accretion rate of $\dot{M} \sim 10^{-4} M_{\odot} \mathrm{yr}^{-1}$. Protostars experiencing high accretion rates develop extended and cool photospheres (Hosokawa \& Omukai 2009). Accretion rates of $\dot{M} \sim 10^{-4}-10^{-3} M_{\odot} \mathrm{yr}^{-1}$ result in photospheric radii reaching values between 0.2 and 0.7 au when the star reaches masses of $5-8 M_{\odot}$. Thus, accreting massive protostars are expected to resemble cool, red supergiants.

Light from radio Source I illuminates a near-infrared reflection nebula extending approximately $1^{\prime}$ east of the OMC1 core. Source I has been indirectly detected at near-IR wavelengths $(3.8$ and $4.7 \mu \mathrm{m})$ through light scattered into our line of sight by this reflection nebula (Sitarski et al. 2013). The spectrum of this nebula indicates that the illuminating source has an effective temperature of $T_{\text {eff }} \approx 4000 \mathrm{~K}$, similar to a K or M supergiant (Morino et al. 1998; Testi et al. 2010). At this effective temperature, the radius of the stellar photosphere is $R \sim 1.5 \times 10^{13} L_{4}^{1 / 2} / T_{\text {eff }}^{2} \mathrm{~cm} \approx 1$ au, where $L_{4}$ is the luminosity in units of $10^{4} L_{\odot}$. The luminosity of the OMC1 core is about $10^{5} L_{\odot}$. Subtracting the contribution from the two known luminous sources, the $\mathrm{BN}$ object $\left(L \sim 1-2 \times 10^{4} L_{\odot}\right)$, and IRc4 $\left(L \sim 2 \times 10^{4} L_{\odot}\right)$ sets an upper bound on the luminosity of radio Source I, of $L_{I} \sim 6 \times 10^{4} L_{\odot}$. Given the effective temperature of Source I, and its luminosity, one can estimate an upper bound on the photospheric radius, $R_{I} \approx\left(L_{I} / 4 \pi \sigma T_{\mathrm{eff}}^{4}\right)^{1 / 2}<3.6 \times 10^{13} \mathrm{~cm}$, or $R_{I}<2.4$ au.

A lower bound on the photospheric radius can be obtained by assuming that the luminosity is entirely due to accretion. Setting the photospheric luminosity equal to the accretion luminosity gives $R \sim\left(G M \dot{M} / 4 \pi \sigma T_{\text {eff }}^{4}\right)^{1 / 2}$, where $M$ is the mass of the protostar, $\dot{M}$ is the accretion rate, $G$ is Newton's gravitational constant, and $\sigma$ is the Stephan-Boltzmann constant. For $T_{\text {eff }}=4000 \mathrm{~K}$, accretion rates of $10^{-5}, 10^{-4}$, and $10^{-3} M_{\odot} \mathrm{yr}^{-1}$ onto a $10 M_{\odot}$ protostar give photospheric radii of $R \sim 0.11,0.24,0.5 \mathrm{au}$, respectively. Note that in a hot core with a gas temperature of $T_{\text {gas }} \sim 400 \mathrm{~K}$, the sound speed is about $c_{s} \sim 1 \mathrm{~km} \mathrm{~s}^{-1}$ in molecular gas. The expected accretion rate (assuming the core is roughly an isothermal sphere) is $\dot{M} \sim c_{s}^{3} / G \sim 3 \times 10^{-4} M_{\odot} \mathrm{yr}^{-1}$. These upper and lower bounds imply that prior to the ejection event $\sim 500$ years ago, massive protostars in the OMC1 core likely had au-scale photospheres. 
If at least one of the stars involved in the interaction 500 years ago was accreting at such a high rate and was bloated, the compact binary configuration required by the energetics implies that the binary semimajor axis is smaller than the photospheric radius of that massive protostar. The companion would have penetrated the bloated star's photosphere where dissipation could have led to a stellar merger.

\subsection{An Interaction-formed Binary or Protostellar Merger?}

The kinetic energy observed in the outflow plus the motion of the ejected stars requires the release of $\sim 10^{48} \mathrm{erg}$. If the interaction produced a compact binary consisting of a pair of $<10 M_{\odot}$ stars, their current mean separation has to be $<2$ au to release this much binding energy. However, if the massive stars in such a binary had bloated photospheres, a mean separation of less than 2 au would have led to a stellar merger. The stellar collision would occur with a velocity comparable to the escape speed from the more massive star's surface, roughly $\sim 100-300 \mathrm{~km} \mathrm{~s}^{-1}$. Because the interacting stars are young, they are unlikely to be tidally locked as is expected to be the case for mature short-period binaries that merge such as V838 Mon (Soker \& Tylenda 2006). Any circumstellar material and the outer stellar atmosphere would be ejected by the collision of photospheres. If a merger occurred, the bulk of the gravitational potential energy release will then occur when the dense stellar cores merge. A $10 M_{\odot}$ protostar can release more than $10^{48} \mathrm{erg}$ required to explain the kinetic energy of stellar motions and the outflow in Orion if the merger partner has a mass larger than about $0.03 M_{\odot}$. Thus, even relatively low-mass merger partners can release sufficient gravitational potential energy to produce the OMC1 outflow.

The disruption of circumstellar disks by close-in $(<1 \mathrm{au})$ stellar encounters would eject debris with speeds comparable to the Kepler speed $\left(>100 \mathrm{~km} \mathrm{~s}^{-1}\right)$. Following the removal of 30 to $40 M_{\odot}$ of stellar mass from the OMC1 core by the ejection of its massive stars, any envelope or circumstellar material surrounding the system that was gravitationally bound to it would expand with speeds ranging from 3 to $30 \mathrm{~km} \mathrm{~s}^{-1}$ if originating from within 1000 to 10 au of the center of mass. In a merger, the bulk of the gravitational potential energy is released by the hypersonic collision of the stellar photospheres followed by the in-spiral of the stellar cores.

Scattered-light images, polarimetry, and direct imaging show that Source I and BN are both surrounded by disks whose major-axis dimensions on the sky are parallel to their respective proper-motion (PM) vectors (Jiang et al. 2005; Reid et al. 2007). Thus the disk spin-axes are at nearly right angles to the PM-vectors. A disk orientation with a spin-axis approximately perpendicular to the velocity vector of an ejected star is expected from angular momentum conservation by fall-back of debris that failed to reach escape speed during the ejection event, or material accreted from the environment by the BondiHoyle process (Bally et al. 2011; Moeckel \& Goddi 2012).

Massive stars with near-Solar abundances of the heavy elements, even ones still forming, fuse hydrogen in their cores. If the stars involved in a merger have masses of more than a few $M_{\odot}$, there could be a nuclear component to energy generation. By the time a star reaches this mass, its core will be fusing hydrogen, mostly by means of the CNO Cycle, which has an incredibly steep temperature dependence with $\dot{E}_{\mathrm{CNO}} \propto T_{\text {core }}^{16}$ (Prialnik 2000). Thus, it is possible that in a stellar merger, perturbations to the pressure and temperature of the core could lead to a dramatic increase in the core luminosity, which could add to the energy of the stellar envelope. Modeling is needed to see how such an event would impact the stellar structure and properties such as its luminosity and surface dynamics to determine if such an event can contribute to the explosion.

The Kelvin-Helmholtz $(\mathrm{K}-\mathrm{H})$ cooling time for a merger remnant, $L \sim G M^{2} / \mathrm{RL}=1400 M_{10}^{2} R_{1}^{-1} L_{4}^{-1}$ years where $M_{10}$ is the remnant mass in units of $10 M_{\odot}, R_{1}$ is its photospheric radius in units of $1 \mathrm{au}$, and $L_{4}$ is its luminosity in units of $10^{4} L_{\odot}$. Thus, a 1 au radius $2 \times 10^{4} L_{\odot}$ object is expected to have a K-H cooling time of $\sim 700$ years. Such a remnant, formed about 500 years ago, would still be expected to resemble a supergiant star. As discussed above, Source I resembles such a high-luminosity, cool object.

\subsection{Explosive Outflows and Infrared-transients}

Orion may not be unique. Spitzer detected $4.5 \mu \mathrm{m}$ emission from a wide-angle outflow emerging from a high-luminosity $\left(\sim 10^{6} L_{\odot}\right)$ hot-core in G34.26+0.15 in the inner Galaxy having a morphology similar to the $\mathrm{H}_{2}$ fingers in Orion (Cyganowski et al. 2008). Unfortunately, this flow and its source are at a distance of $\sim 5 \mathrm{kpc}$ and highly obscured. Source G in W49, the most luminous water maser outflow in the Milky Way, may be another example (Smith et al. 2009). Interstellar bullets similar to the OMC1 fingers emerge from the massive protostar IRAS $05506+2414$ (Sahai et al. 2008) and from DR21 (Zapata et al. 2013).

In high-density, cluster-forming environments such as Orion, where protostellar number densities can exceed $10^{4}-10^{5}$ stars per cubic parsec, encounters between massive stars with bloated photospheres and other cluster members are likely to be relatively common. Such encounters are facilitated by the dissipative nature of dense cloud cores, which drain orbital angular momentum rapidly from the most massive protostars, causing them to be dragged into the center of the gravitational potential well of the star-forming region. The high multiplicity among massive stars may be a consequence of such interactions (Moeckel \& Bally 2007a) and may promote violent interactions between binaries and single stars or other binaries (Goddi et al. 2011b) that may lead to mergers. Orionlike events may thus be relatively common in massive starforming complexes, occurring one or more times during the birth of a massive star. Their shock-generated signatures would be erased on a timescale required for the shocks to cross the dimensions of the cloud core, $\sim 10^{3}$ to $\sim 10^{4}$ years. However, such events may produce luminous infrared flares (Bally \& Zinnecker 2005) and may be a major source of transient luminosity, kinetic energy, and momentum feedback in the self-regulation of star formation in regions forming dense star clusters and massive stars.

Smith et al. (2016) found a luminous visual transient, NGC 4490-OT, in the galaxy NGC 4490. This object is a candidate massive stellar merger event similar to but more luminous than V838 Mon. The Spitzer Space Telescope during its warm mission phase found over 50 luminous transient events in galaxies located within $20 \mathrm{Mpc}$ at 3.6 and $4.5 \mu \mathrm{m}$ but with no counterparts in visual or near-IR images (Jencson et al. 2016a, 2016b; Kasliwal et al. 2016). Most are found in the dusty spiral arms of star-forming galaxies such as M83. These objects have luminosities intermediate between novae and supernovae. Although some may be supernovae, ultra- 
luminous novae hidden behind dense clouds, or self-obscured within their own envelopes, the number of these luminous IRtransients suggests that they trace other types of eruptive phenomena such as stellar mergers in compact binary systems, eta-Carina type events, or Orion-like eruptions.

\section{Conclusions}

We present new observations of the Orion OMC1 outflow in the $230 \mathrm{GHz} J=2-1 \mathrm{CO}$ line with a resolution of $\sim 1^{\prime \prime}$. The new data confirm the explosive nature of the outflow. The main results of this study are as follows.

1. The high-velocity $\mathrm{CO}$ emission consists of over 100 linear streamers of $\mathrm{CO}$ emission extending to radial velocities of $\pm 100 \mathrm{~km} \mathrm{~s}^{-1}$. The streamers exhibit a roughly isotropic, spherically symmetric distribution exhibiting a "Hubble flow" with the radial velocity proportional to the projected distance from the explosion center. The linear velocity-distance relation implies that the ejecta are orders of magnitude denser than the medium through which they move. They tend to be brightest at their leading edges.

2. Toward the northeast and southwest, the CO streamers nearly reach the locations of the $\mathrm{H}_{2}$ and [Fe II] shocks. Furthermore, the range of $\mathrm{CO}$ radial velocities is comparable to the range of values of the $\mathrm{H}_{2}$ proper motions when the fastest [Fe II] features and $\mathrm{HH}$ objects are excluded.

3. Toward the north and northwest, the bright $T_{B}>10 \mathrm{~K}$ $\mathrm{CO}$ streamers have a projected spatial extent of about a factor of two smaller than the extent of the outflow as traced by $\mathrm{H}_{2}$ and [Fe II]. No CO clumps or bullets are seen at the leading edges of the north or northwest oriented fingers down to brightness temperatures of the order of $0.1 \mathrm{~K}$. A few of the north and northwest fingers of $\mathrm{H}_{2}$ contain faint clumps of low-radial velocity $\mathrm{CO}$ along most of their lengths, many of which are associated with $\mathrm{H}_{2}$ and [Fe II] bow shocks. However, the two brightest [Fe II] shocks, which are associated with HH 201 and HH 210 have no clear CO streamers aimed at them. The highest red- and blueshifted radial velocities are about a factor of two smaller than the fastest proper motions measured in these near-IR lines. These features suggest that most of the ejecta toward the north or northwest iare moving very close to the plane of the sky. However, it is also possible that shocks dissociate accelerated molecular gas, that the gas was launched as atomic or ionized material and did not form molecules, or there is a density gap between the shocks traced by nearIR emission lines and the ends of the CO streamers.

4. Several limb-brightened bubbles of ejecta emerge from the OMC1 region at velocities within tens of $\mathrm{km} \mathrm{s}^{-1}$ of the line core. The most prominent bubbles are the redshifted feature extending to the east and the blueshifted bubble extending toward the southeast whose southern rim lies behind the Trapezium. A less coherent bubble of both red- and blueshifted gas extends toward the northwest.

5. Deviations from spherical symmetry provide clues about the origin of the OMC1 explosion. High-velocity ejecta extend toward the northwest, and bubble-like features extending toward the east and southeast that may represent streams of dynamically ejected debris launched in a sub-au-scale encounter of three or more stars. The previously detected young, thermal $\mathrm{SiO}$ and $\mathrm{H}_{2} \mathrm{O}$ maser flow emerging from Source I contains the brightest and highest density portion of the OMC1 outflow.

6 . The $\sim 500$ year dynamical age of the outflow coincides with the time when radio Source I, the BN object, and Source $\mathrm{n}$ were closest to each other. The coincidence between the age of the outflow and the time of closest approach of the stars suggests that both the outflow and the stars were ejected from OMC1 by a dynamical interaction of four or more protostars. It is also possible that the initial interaction with $\mathrm{BN}$, produced an unstable system that decayed up to a few hundred years later and launched Source I and Source $\mathrm{n}$ on their current trajectories. Such a staged disintegration could explain the several arcsecond dispersion in streamer orientations.

7. The energy of the event was likely powered by the dynamical interaction of a group of stars in the OMC1 core that led to either the formation of a compact (auscale) binary containing a pair of massive stars, or the dynamically induced merger of two stars. Such an event may have produced an infrared-only flare with a luminosity between novae and supernovae and a duration of years to decades.

The OMC1 explosive outflow and stellar ejection poses many puzzles. Is the mass of Source I as low as suggested by Plambeck \& Wright (2016)? If so, how is the momentum of the $\mathrm{BN}$ object, which is moving toward the northwest, balanced by stars ejected in the opposite direction? Are there additional ejected stars, or compact masses such as nascent protostars like IRc4 involved? Was the mass in the hot core or other compact clumps involved in the dynamic ejection? Or, is the Source I disk in sub-Keplerian rotation, possibly because of internal pressure support provided by magnetic fields, turbulence, or perturbations associated fall-back and dynamic ejection a halfmillennium ago? How were the hundreds of $\mathrm{CO}$ streamers produced? The $\mathrm{CO}$ streamers do not reach the most distant $\mathrm{HH}$ objects, $\mathrm{H}_{2}$, and [Fe II] shocks in the OMC1 BN/KL outflow. What continues to drive the fast motions of these shocks? How common are OMC1-like explosive outflows? How much do such events contribute to feedback and self-regulation of star formation?

This work was supported in part by National Science Foundation (NSF) grant AST-1009847. This paper uses ALMA data obtained with program ADS/JAO.ALMA \#2013.1.00546.S. ALMA is a partnership of the European Southern Observatory (ESO) representing member states, Associated Universities Incorporated (AUI) and the National radio Astronomy Observatories (NRAO) for the National Science Foundation (NSF) in the USA, NINS in Japan, NRC in Canada, and NSC and ASIAA in Taiwan, in cooperation with the Republic of Chile. The Joint ALMA Observatory (JAO) is operated by ESO (Europe), AUI/NRAO (USA), and NAOJ (Japan). L.A.Z. is grateful to CONACyT, Mexico, and DGAPA, UNAM for their financial support. J.B. thanks Professor Andreas Burkert from the Ludwig-Maximilians Universitat Munchen, Department fur Physik, for insightful discussion and for producing numerical simulations of streamer formation and Professor Luis F. Rodrigues for sharing the 
results of recent radio proper motion measurements of sources in OMC1.

Facility: ALMA.

Software: IRAF, ds9, CASA (McMullin et al. 2007), RADEX (van der Tak et al. 2007).

\section{References}

Allen, D. A., \& Burton, M. G. 1993, Natur, 363, 54

Axon, D. J., \& Taylor, K. 1984, MNRAS, 207, 241

Bally, J. 2016, ARA\&A, 54, 491

Bally, J., Cunningham, N. J., Moeckel, N., et al. 2011, ApJ, 727, 113

Bally, J., Ginsburg, A., Silvia, D., \& Youngblood, A. 2015, A\&A, 579, A130

Bally, J., Langer, W. D., Stark, A. A., \& Wilson, R. W. 1987, ApJL, 312, L45

Bally, J., \& Reipurth, B. 2001, ApJ, 546, 299

Bally, J., \& Zinnecker, H. 2005, AJ, 129, 2281

Bolatto, A. D., Wolfire, M., \& Leroy, A. K. 2013, ARA\&A, 51, 207

Chatterjee, S., \& Tan, J. C. 2012, ApJ, 754, 152

Colgan, S. W. J., Schultz, A. S. B., Kaufman, M. J., Erickson, E. F., \& Hollenbach, D. J. 2007, ApJ, 671, 536

Crutcher, R. M. 2012, ARA\&A, 50, 29

Cyganowski, C. J., Whitney, B. A., Holden, E., et al. 2008, AJ, 136, 2391

De Buizer, J. M., Morris, M. R., Becklin, E. E., et al. 2012, ApJL, 749, L23

Doi, T., O'Dell, C. R., \& Hartigan, P. 2002, AJ, 124, 445

Doi, T., O'Dell, C. R., \& Hartigan, P. 2004, AJ, 127, 3456

Dray, L. M., Dale, J. E., Beer, M. E., Napiwotzki, R., \& King, A. R. 2005, MNRAS, 364, 59

Dzib, S. A., Loinard, L., Rodríguez, L. F., et al. 2017, ApJ, 834, 139

Frank, A., Ray, T. P., Cabrit, S., et al. 2014, in Protostars and Planets VI, ed. H. Beuther et al. (Tucson, AZ: Univ. Arizona Press), 451

Frost, D. L., Grégoire, Y., Petel, O., Goroshin, S., \& Zhang, F. 2012, PhFl, 24, 091109

Genzel, R., Reid, M. J., Moran, J. M., \& Downes, D. 1981, ApJ, 244, 884

Genzel, R., \& Stutzki, J. 1989, ARA\&A, 27, 41

Gies, D. R., \& Bolton, C. T. 1986, ApJS, 61, 419

Girart, J. M., Greaves, J. S., Crutcher, R. M., \& Lai, S.-P. 2004, Ap\&SS, 292, 119

Goddi, C., Greenhill, L. J., Humphreys, E. M. L., Chandler, C. J., \& Matthews, L. D. 2011a, ApJL, 739, L13

Goddi, C., Humphreys, E. M. L., Greenhill, L. J., Chandler, C. J., \& Matthews, L. D. 2011b, ApJ, 728, 15

Goicoechea, J. R., Chavarría, L., Cernicharo, J., et al. 2015, ApJ, 799, 102

Gómez, L., Rodríguez, L. F., Loinard, L., et al. 2008, ApJ, 685, 333

Graham, M. F., Meaburn, J., \& Redman, M. P. 2003, MNRAS, 343, 419

Greenhill, L. J., Gezari, D. Y., Danchi, W. C., et al. 2004, ApJL, 605, L57

Greenhill, L. J., Gwinn, C. R., Schwartz, C., Moran, J. M., \& Diamond, P. J. 1998, Natur, 396, 650

Grosso, N., Feigelson, E. D., Getman, K. V., et al. 2006, A\&A, 448, L29

Gualandris, A., Portegies Zwart, S., \& Eggleton, P. P. 2004, MNRAS, 350, 615

Gvaramadze, V. V., \& Gualandris, A. 2011, MNRAS, 410, 304

Hoogerwerf, R., de Bruijne, J. H. J., \& de Zeeuw, P. T. 2000, ApJL, 544, L133

Hoogerwerf, R., de Bruijne, J. H. J., \& de Zeeuw, P. T. 2001, A\&A, 365, 49

Hosokawa, T., \& Omukai, K. 2009, ApJ, 691, 823

Jencson, J. E., Kasliwal, M. M., Tinyanont, S., et al. 2016a, ATel, 8688

Jencson, J. E., Kasliwal, M. M., Tinyanont, S., et al. 2016b, ATel, 8940

Jiang, Z., Tamura, M., Fukagawa, M., et al. 2005, Natur, 437, 112

Johnstone, D., \& Bally, J. 1999, ApJL, 510, L49

Jones, B. F., \& Walker, M. F. 1985, AJ, 90, 1320

Kaifu, N., Usuda, T., Hayashi, S. S., et al. 2000, PASJ, 52, 1

Kasliwal, M. M., Bally, J., Masci, F., et al. 2016, ApJ, in press (arXiv:1701. 01151)

Kwan, J., \& Scoville, N. 1976, ApJL, 210, L39
Lee, J.-K., \& Burton, M. G. 2000, MNRAS, 315, 11

Lonsdale, C. J., Becklin, E. E., Lee, T. J., \& Stewart, J. M. 1982, AJ, 87, 1819

Luhman, K. L., Rieke, G. H., Young, E. T., et al. 2000, ApJ, 540, 1016

Matthews, L. D., Greenhill, L. J., Goddi, C., et al. 2010, ApJ, 708, 80

McCaughrean, M. J., \& Mac Low, M.-M. 1997, AJ, 113, 391

McMullin, J. P., Waters, B., Schiebel, D., Young, W., \& Golap, K. 2007, in ASP Conf. Ser. 376, Astronomical Data Analysis Software and Systems XVI, ed. R. A. Shaw, F. Hill, \& D. J. Bell (San Francisco, CA: ASP), 127 Menten, K. M., \& Reid, M. J. 1995, ApJL, 445, L157

Menten, K. M., Reid, M. J., Forbrich, J., \& Brunthaler, A. 2007, A\&A, 474,515

Milne, A., Longbottom, A., Frost, D. L., et al. 2016, ShWav

Moeckel, N., \& Bally, J. 2007a, ApJ, 656, 275

Moeckel, N., \& Bally, J. 2007b, ApJL, 661, L183

Moeckel, N., \& Goddi, C. 2012, MNRAS, 419, 1390

Morino, J.-I., Yamashita, T., Hasegawa, T., \& Nakano, T. 1998, Natur, 393, 340

O’Dell, C. R., Ferland, G. J., Henney, W. J., et al. 2015, AJ, 150, 108

O'dell, C. R., Hartigan, P., Bally, J., \& Morse, J. A. 1997a, AJ, 114, 2016

O'dell, C. R., Hartigan, P., Lane, W. M., et al. 1997b, AJ, 114, 730

Oh, H., Pyo, T.-S., Kaplan, K. F., et al. 2016, arXiv:1610.09459

Plambeck, R. L., Bolatto, A. D., Carpenter, J. M., et al. 2013, ApJ, 765, 40

Plambeck, R. L., \& Wright, M. C. H. 2016, arXiv:1608.00996

Plambeck, R. L., Wright, M. C. H., Friedel, D. N., et al. 2009, ApJL, 704, L25

Plambeck, R. L., Wright, M. C. H., \& Rao, R. 2003, ApJ, 594, 911

Portegies Zwart, S. F., \& van den Heuvel, E. P. J. 2016, MNRAS, 456, 3401

Prialnik, D. 2000, An Introduction to the Theory of Stellar Structure and Evolution (Cambridge: Cambridge Univ. Press)

Reid, M. J., Menten, K. M., Greenhill, L. J., \& Chandler, C. J. 2007, ApJ, 664,950

Reipurth, B., \& Bally, J. 2001, ARA\&A, 39, 403

Reipurth, B., \& Mikkola, S. 2012, Natur, 492, 221

Reipurth, B., \& Mikkola, S. 2015, AJ, 149, 145

Reipurth, B., Mikkola, S., Connelley, M., \& Valtonen, M. 2010, ApJL, 725, L56

Sahai, R., Claussen, M., Sánchez Contreras, C., Morris, M., \& Sarkar, G. 2008, ApJ, 680, 483

Scoville, N., Kleinmann, S. G., Hall, D. N. B., \& Ridgway, S. T. 1983, ApJ, 275,201

Shu, F. H., Lizano, S., Galli, D., Cai, M. J., \& Mohanty, S. 2008, ApJL, 682, L121

Sitarski, B. N., Morris, M. R., Lu, J. R., et al. 2013, ApJ, 770, 134

Smith, N., Andrews, J. E., Van Dyk, S. D., et al. 2016, MNRAS, 458, 950

Smith, N., Whitney, B. A., Conti, P. S., de Pree, C. G., \& Jackson, J. M. 2009, MNRAS, 399, 952

Snell, R. L., Scoville, N. Z., Sanders, D. B., \& Erickson, N. R. 1984, ApJ, 284, 176

Soker, N., \& Tylenda, R. 2006, MNRAS, 373, 733

Stone, J. M., Xu, J., \& Mundy, L. G. 1995, Natur, 377, 315

Tan, J. C. 2004, ApJL, 607, L47

Tauris, T. M., \& Takens, R. J. 1998, A\&A, 330, 1047

Teixeira, P. S., Takahashi, S., Zapata, L. A., \& Ho, P. T. P. 2016, A\&A, 587, A47

Testi, L., Tan, J. C., \& Palla, F. 2010, A\&A, 522, A44

van der Tak, F. F. S., Black, J. H., Schöier, F. L., Jansen, D. J., \& van Dishoeck, E. F. 2007, A\&A, 468, 627

Youngblood, A., Ginsburg, A., \& Bally, J. 2016, AJ, 151, 173

Zapata, L. A., Loinard, L., Schmid-Burgk, J., et al. 2011a, ApJL, 726, L12

Zapata, L. A., Rodríguez, L. F., Schmid-Burgk, J., et al. 2012, ApJL, 754, L17

Zapata, L. A., Schmid-Burgk, J., Ho, P. T. P., Rodríguez, L. F., \& Menten, K. M. 2009, ApJL, 704, L45

Zapata, L. A., Schmid-Burgk, J., \& Menten, K. M. 2011b, A\&A, 529, A24

Zapata, L. A., Schmid-Burgk, J., Pérez-Goytia, N., et al. 2013, ApJL, 765 L29 